\title{
Ramification groups in Artin-Schreier-Witt extensions
}

\author{
par LARA THOMAS
}

\begin{abstract}
RÉSUMÉ. Soit $K$ un corps local de caractéristique $p>0$. L'objectif de cet article est de décrire les groupes de ramification des pro- $p$ extensions abéliennes de $K$ à travers la théorie d'Artin-SchreierWitt. Dans le cadre usuel de la théorie du corps de classes local, cette étude est menée entièrement et conduit à un accouplement non-dégénéré que nous définissons en détail, généralisant ainsi la formule de Schmid pour les vecteurs de Witt de longueur $n$. Au passage, on retrouve un résultat de Brylinski avec des arguments plus explicites nécessitant moins d'outils techniques. La dernière partie aborde le cas plus général où le corps résiduel de $K$ est parfait.
\end{abstract}

Abstract. Let $K$ be a local field of characteristic $p>0$. The aim of this paper is to describe the ramification groups for the pro$p$ abelian extensions over $K$ with regards to the Artin-SchreierWitt theory. We shall carry out this investigation entirely in the usual framework of local class field theory. This leads to a certain non-degenerate pairing that we shall define in detail, generalizing in this way the Schmid formula to Witt vectors of length $n$. Along the way, we recover a result of Brylinski but with a different proof which is more explicit and requires less technical machinery. A first attempt is finally made to extend these computations to the case where the perfect field of $K$ is merely perfect.

\section{Introduction}

By a local field we mean a discrete valuation field with perfect residue field. Let $p$ be a prime number. This paper is concerned with the ramification groups for the Artin-Schreier-Witt extensions over a local field $K$ of characteristic $p$, i.e. for its pro- $p$ abelian extensions.

We fix once and for all a separable closure $K^{\text {sep }}$ of $K$. For each $n \geq 1$, let $G_{p^{n}}$ be the Galois group of the maximal abelian extension of exponent $p^{n}$ over $K$ and let $G_{p^{\infty}}$ be the Galois group of the maximal pro- $p$

Manuscrit reçu le 29 juillet 2005. 
abelian extension over $K$. We also denote by $W(K)$ the commutative ring of Witt vectors over $K$ and by $W_{n}(K)$ the quotient ring of truncated Witt vectors of length $n$. We shall consider the additive group morphism $\wp: W_{n}(K) \rightarrow W_{n}(K)$ given by $\wp\left(x_{0}, x_{1}, \ldots\right)=\left(x_{0}^{p}, x_{1}^{p}, \ldots\right)-\left(x_{0}, x_{1}, \ldots\right)$. Its kernel is $W_{n}\left(\mathbb{F}_{p}\right) \simeq \mathbb{Z} / p^{n} \mathbb{Z}$.

The Artin-Schreier-Witt theory [17] yields isomorphisms of topological groups:

$$
\begin{array}{cccc}
\mathfrak{a s}_{n}: \quad G_{p^{n}} & \stackrel{\simeq}{\longrightarrow} & H_{p^{n}}=\operatorname{Hom}\left(W_{n}(K) / \wp\left(W_{n}(K)\right), W_{n}\left(\mathbb{F}_{p}\right)\right) \\
\sigma & \mapsto & \varphi_{\sigma}:\left\{a+\wp\left(W_{n}(K)\right) \mapsto \sigma(\alpha)-\alpha\right\}
\end{array}
$$

for some $\alpha \in W_{n}\left(K^{\text {sep }}\right)$ such that $\wp(\alpha)=a$ and where the action of $G_{p^{n}}$ on $W_{n}\left(K^{\text {sep }}\right)$ is defined componentwise. Here, $H_{p^{n}}$ is provided with the product topology induced by the discrete topology on $W_{n}\left(\mathbb{F}_{p}\right)$.

The goal of this paper is, given $n \geq 1$, to describe explicitly how the ramification groups $G_{p^{n}}^{(u)}$ of $G_{p^{n}}$ in the upper numbering behave under the Artin-Schreier-Witt isomorphism $\mathfrak{a s}_{n}$.

When the residue field of $K$ is finite, the interplay between local class field theory and Artin-Schreier-Witt theory gives rise to a non-degenerate pairing, that we shall call the Artin-Schreier-Witt symbol:

$$
\begin{array}{cl}
W_{n}(K) / \wp\left(W_{n}(K)\right) \times K^{*} / K^{* p^{n}} & \rightarrow W_{n}\left(\mathbb{F}_{p}\right) \\
\left(a+\wp\left(W_{n}(K)\right), b . K^{* p^{n}}\right) & \mapsto[a, b):=(b, L / K)(\alpha)-\alpha,
\end{array}
$$

where $\wp(\alpha)=a, L=K(\alpha)$ and $(b, L / K)$ is the norm residue symbol of $b$ in $L / K$. We shall compute this symbol explicitly so as to get the ramification groups of $G_{p^{n}}$ by Pontryagin duality under the existence theorem.

Our main result is then the following:

Theorem 1.1. Let $K$ be a local field of characteristic $p$ with finite residue field. For every integer $u \geq 0$, the Artin-Schreier-Witt isomorphism $\mathfrak{a s}_{n}$ induces isomorphisms of topological groups:

$$
G_{p^{n}}^{(0)} \stackrel{\simeq}{\longrightarrow} H_{p^{n}}^{(0)} \text { and } G_{p^{n}}^{(u)} \stackrel{\simeq}{\longrightarrow} H_{p^{n}}^{(u-1)} \text { if } u \geq 1 .
$$

Here, for each integer $v \geq-1, H_{p^{n}}^{(v)}$ is the subgroup of $H_{p^{n}}$ given by:

$$
H_{p^{n}}^{(v)}:=\left\{\varphi \in H_{p^{n}}: \varphi\left(W_{n}^{(v)}(K)+\wp\left(W_{n}(K)\right) / \wp\left(W_{n}(K)\right)\right)=0\right\},
$$

where we set:

$$
W_{n}^{(v)}(K):=\left(\mathfrak{p}_{K}^{-\left\lfloor\frac{v}{p^{n-1}}\right\rfloor}, \mathfrak{p}_{K}^{-\left\lfloor\frac{v}{p^{n-2}}\right\rfloor}, \cdots, \mathfrak{p}_{K}^{-v}\right)
$$


For all $v \geq-1$, the $W_{n}^{v}(K)$ form an increasing sequence of subgroups of $W_{n}(K)$, with $W_{n}^{0}(K)=W_{n}\left(O_{K}\right)$ and $W_{n}^{-1}(K)=\left(\mathfrak{p}_{k}, \ldots, \mathfrak{p}_{K}\right)$. The groups $H_{p^{n}}^{(v)}$ form an exhaustive and decreasing filtration of $H_{p^{n}}$ that theorem 1.1 puts in bijection with the ramification groups of $G_{p^{n}}$.

By taking the inverse limit when $n$ tends to infinity, we get an ArtinSchreier-Witt isomorphim for the Galois group $G_{p^{\infty}}$ of the maximal pro- $p$ abelian extension over $K$ :

$$
\begin{array}{cccc}
\mathfrak{a s}_{\infty}: G_{p^{\infty}} & \stackrel{\simeq}{\longrightarrow} & H_{p^{\infty}}=\operatorname{Hom}\left(W(K) / \wp(W(K)), W\left(\mathbb{F}_{p}\right)\right) \\
\sigma & \mapsto & \varphi_{\sigma}:\{a+\wp(W(K)) \mapsto \sigma(\alpha)-\alpha\}
\end{array}
$$

where $W\left(\mathbb{F}_{p}\right) \simeq \mathbb{Z}_{p}$ is provided with the $p$-adic topology. Then, we may deduce from theorem 1.1 the ramification groups for $G_{p^{\infty}}$ :

Corollary 1.1. The Artin-Schreier-Witt isomorphism $\mathfrak{a s}_{\infty}$ gives rise to isomorphisms of topological groups:

$$
G_{p^{\infty}}^{(0)} \stackrel{\simeq}{\longrightarrow} \bigcap_{n \geq 1} H_{p^{\infty}}^{(0, n)} \text { and } G_{p^{\infty}}^{(u)} \stackrel{\simeq}{\longrightarrow} \bigcap_{n \geq 1} H_{p^{\infty}}^{(u-1, n)} \text { if } u \geq 1,
$$

where for all $n \geq 1$ and for all $u \geq-1$ :

$$
\begin{aligned}
H_{p^{\infty}}^{(u, n)}:=\{\varphi \in & H_{p^{\infty}}, \\
& \varphi\left(\left(\mathfrak{p}_{K}^{-\left\lfloor\frac{u}{p^{n-1}}\right\rfloor}, \ldots, \mathfrak{p}_{K}^{-u}, *, \ldots\right) \bmod \wp(W(K)) \subset V^{n} W\left(\mathbb{F}_{p}\right)\right\} .
\end{aligned}
$$

Another consequence of theorem 1.1 is concerned with the computation of the Artin conductor for a cyclic extension of degree $p^{n}$ over $K$ (see corollary 5.1 of section 5). In this manner, we refine Satz 3 of [12] by writing explicitly every crucial step of the proof and in particular by describing precisely the Schmid-Witt residue formula for Witt vectors of length $n$ in characteristic $p$. At the same time, we obtain a more direct and more explicit proof for Brylinski's Theorem 1 of [2]. We may also mention [4] and we thank Michel Matignon for pointing out this reference.

One question is then to tackle the problem when the residue field of $K$ is assumed to be merely perfect and we partially solve it in two directions: we show that in this context theorem 1.1 is still valid for all ramification groups of $G_{p}$ as well as for the inertia group of each $G_{p^{n}}, n \geq 1$. Indeed, completing [7] we first claim: 
Theorem 1.2. Let $K$ be a local field of characteristic $p$ with perfect residue field. The Artin-Schreier isomorphism $\mathfrak{a s}_{1}$ induces isomorphisms of topological groups:

$$
G_{p}^{(0)} \stackrel{\simeq}{\longrightarrow} H_{p}^{(0)} \text { and } \quad G_{p}^{(u)} \stackrel{\simeq}{\longrightarrow} H_{p}^{(u-1)} \text { if } u \geq 1 .
$$

As for the inertia group of every maximal abelian extension of exponent $p^{n}$ over $K$, we shall finally state:

Theorem 1.3. Let $K$ be a local field of characteristic $p$ with perfect residue field. For each $n \geq 1$, the Artin-Schreier-Witt isomorphism $\mathfrak{a s}_{n}$ induces on the inertia group of $G_{p^{n}}$ the isomorphism of topological groups:

$$
G_{p^{n}}^{(0)} \stackrel{\simeq}{\longrightarrow}\left\{\varphi \in H_{p^{n}} ; \varphi\left(W_{n}\left(O_{K}\right) / \wp\left(W_{n}\left(O_{K}\right)\right)\right)=0\right\} .
$$

Passing to the inverse limit, $\mathfrak{a s}_{\infty}$ yields an isomorphism of topological groups:

$$
G_{p^{\infty}}^{(0)} \stackrel{\simeq}{\longrightarrow}\left\{\varphi \in H_{p^{\infty}} ; \varphi\left(W\left(O_{K}\right) / \wp\left(W\left(O_{K}\right)\right)\right)=0\right\} .
$$

where $O_{K}$ denotes the valuation ring of $K$.

One may notice that this last theorem corroborates theorem 1.1 for $u=0$ and for all $n \geq 1$ since $H_{p^{n}}^{(0)}=\left\{\varphi \in H_{p^{n}}: \varphi\left(W_{n}\left(O_{K}\right) / \wp\left(W_{n}\left(O_{K}\right)\right)\right)=0\right\}$ because $\wp\left(W_{n}\left(O_{K}\right)\right)=W_{n}\left(O_{K}\right) \cap \wp\left(W_{n}(K)\right)$. Moreover, its proof will also show that it corroborates corollary 1.1 for $u=0$.

When $K$ has finite residue field, the key point is that the Artin-SchreierWitt symbol is non-degenerate and that it can be expressed as the trace of an explicit symbol, the Schmid-Witt symbol which is actually a "residue Witt vector". This reciprocity formula was first discovered by Schmid [10] in 1936 for the local norm symbol of cyclic extensions of degree $p$ in characteristic $p$, following up the work initiated by Schmidt (with a " $t$ ") and then Hasse to establish class field theory for function fields. Then, Witt [17] generalized Schmid's formula to cyclic extensions of degree $p^{n}$. The formula is now classical, but unfortunately not as well known as it deserves to be and Witt actually proved it in characteristic 0 only. For these reasons, in this paper, an attempt is made to develop this norm residue formula for Witt vectors of any length over $K$ very carefully, sparing none of the details.

When the residue field of $K$ is perfect, the crucial step is proposition 6.2 which describes ramification groups for a compositum. Note that we broach the topic from two different approaches: when the residue field of $K$ is finite, local class field theory allows us to directly get the ramification for maximal 
Artin-Schreier-Witt extensions, but when it is merely perfect we first need to compute the ramification groups for finite abelian extensions of exponent dividing $p^{n}$ before taking the compositum.

This note is organized as follows. We start with a preliminary section of brief reminders in ramification theory and local class field theory for the convenience of the reader but also to define the setup for the sequel. This section also provides some terminology for Witt vectors. Then section 3 is the technical heart of the paper since it deals with the Schmid-Witt residue formula for Witt vectors of length $n$ when $K$ has finite residue field. Next section 4 develops the notion of reduced Witt vectors; this is used to compute the Artin-Schreier-Witt symbol in section 5 and thus to describe the ramification groups for all maximal abelian extensions of exponent $p^{n}$ over $K$, proving theorem 1.1 this way. Finally, section 6 is a first approach to the analogous problem when the residue field of $K$ is perfect with a direct proof for theorems 1.2 and 1.3.

Acknowledgments. I would like to thank Hendrik W. Lenstra for many motivating and fruitful discussions and I am indebted to Farshid Hajir for his suggestions in the writing of this paper. I am also grateful to the GTEM network and in particular to the node of Bordeaux for having supported me during my several stays at the University of Leiden.

The present paper is mainly issued from my Ph.D. thesis [16] that I did at the University of Toulouse II under the direction of Christian Maire (Toulouse) and Bart de Smit (Leiden).

\section{Preliminaries}

This section collects some definitions and standard properties of higher ramification groups as well as some terminology from local class field theory. For more details, we shall refer the reader to [13], from which we borrow most of the notation in this paper. In addition, we shall also describe the basic problems we are concerned with in this paper.

Throughout, we will use the following notations: $O_{K}$ is the valuation ring of $K, \mathfrak{p}_{K}$ its maximal ideal, $U_{K}$ is the unit group of $K$ and $\kappa$ its residue field. Once we choose a uniformizing element $T$ for $K$, we may identify $K$ with the field $\kappa((T))$ of formal power series over $\kappa$. When $\kappa$ is supposed to be finite, we will write $\kappa=\mathbb{F}_{q}$ for some power $q$ of $p$. 
Recall that if $L / K$ is a finite abelian extension of Galois group $G$, the $i$ th ramification groups in the lower numbering are defined as:

$$
G_{(i)}:=\left\{\sigma \in G: \forall x \in O_{L}, \sigma(x)-x \in \mathfrak{p}_{L}^{i+1}\right\}
$$

for all integers $i \geq-1$. They form a decreasing filtration of $G$ : $G_{(-1)}=G \supset$ $G_{(0)} \supset G_{(1)} \supset \ldots \supset G_{(m)}=0$, for some $m \geq 0$. If $v \geq-1$ is a real number, we set $G_{(v)}=G_{\left(i_{v}\right)}$ where $i_{v}$ is the only integer such that $i_{v}-1<v \leq i_{v}$. The problem is that these ramification groups are not adapted to quotients and thus they cannot be extended to infinite extensions. Now, there exists a homeomorphism $\psi:[-1 ;+\infty[\rightarrow[-1 ;+\infty[$, usually called the Herbrand function, such that if one sets:

$$
\forall u \geq-1, u \in \mathbb{R}, G^{(u)}:=G_{\psi(u)}
$$

the groups $G^{(u)}$ not only form a decreasing filtration in $G$ again:

$$
G^{(-1)}=G \supset G^{(0)} \supset G^{(1)} \supset \ldots \supset G^{(l)}=0,
$$

with $l \geq 0$, but also they are adapted to quotients in the sense that for all normal subgroups $H$ of $G$ one gets:

$$
\forall u \geq-1,(G / H)^{(u)}=G^{(u)} H / H .
$$

This relation is often called the Herbrand theorem. We can thus define ramification groups in the upper numbering for an infinite abelian extension by taking the inverse limit over all finite subextensions.

In the case of a finite Artin-Schreier-Witt extension, one has $G^{(1)}=G^{(0)}$ because $L / K$ is wildly ramified. Taking the inverse limit, this remains true if $L / K$ is infinite. Moreover, it is only in the wildly ramified case that nontrivial $G^{(u)}$ 's occur for $u \geq 1$, hence our motivation to investigate higher ramification groups in the Artin-Schreier-Witt extensions.

For a cyclic extension of degree $p$ over $K$, following [5] it is well known that:

Proposition 2.1. Let $K$ be a local field of characteristic $p>0$ with perfect residue field. Let $L / K$ be the extension given by the equation $X^{p}-X=a$ for some $a \in K$ and denote by $G$ its Galois group. Then we have:

(1) If $v_{K}(a)>0$ or if $v_{K}(a)=0$ and $a \in \wp(K)$, the extension $L / K$ is trivial.

(2) If $v_{K}(a)=0$ and if $a \notin \wp(K)$, the extension $L / K$ is cyclic of degree $p$ and unramified.

(3) If $v_{K}(a)=-m<0$ with $m \in \mathbb{Z}_{>0}$ and if $m$ is prime to $p$, the extension $L / K$ is cyclic of degree $p$ again and totally ramified. Moreover, 
its ramification groups are given by:

$$
G=G^{(-1)}=\ldots=G^{(m)} \text { and } G^{(m+1)}=1 .
$$

This list is exhaustive. Indeed, if $v_{K}(a)<0$ and if $p$ divides $v_{K}(a)$, then modulo $\wp\left(W_{n}(K)\right), a$ is congruent to some $b \in W_{n}(K)$ such that $v_{K}(b)>v_{K}(a)$ since the residue field of $K$ is perfect. The extension $L$ is also defined by $X^{p}-X=b$ and by iteration we are reduced to one of the previous cases. When $\kappa$ is finite, this argument can be generalised easily to every extension of exponent $p^{n}$ over $K$ for which no jump in the filtration of its ramification groups is divisible by $p^{n}$ (see section 5 ).

To a cyclic extension $L / K$ of degree $p^{n}$, Artin-Schreier-Witt theory attaches a Witt vector $a \in W_{n}(K)$ modulo $\wp\left(W_{n}(K)\right)$ such that $L=$ $K\left(\alpha_{0}, \ldots, \alpha_{n-1}\right)$ where $\alpha=\left(\alpha_{0}, \ldots, \alpha_{n-1}\right) \in W_{n}\left(K^{\text {sep }}\right)$ and $\wp(\alpha)=a$. Thereby, as a corollary of proposition 2.1 , we already have:

Corollary 2.1. Let $L / K$ be a cyclic extension of degree $p^{n}$ given by some Witt vector $a=\left(a_{0}, \ldots, a_{n-1}\right)$ in $W_{n}(K)$. If all components of a lie in $O_{K}$, then $L / K$ is unramified.

Proof. Let $\alpha=\left(\alpha_{0}, \ldots, \alpha_{n-1}\right) \in W_{n}\left(K^{\text {sep }}\right)$ be such that $\wp(\alpha)=a$. For all $i \in\{0, \ldots, n-1\}$, write $K_{i}=K\left(\alpha_{0}, \ldots, \alpha_{i}\right)$ and $K_{-1}=K$. Then by iteration each extension $K_{i} / K_{i-1}$ is cyclic of degree $p$ and it is given by an equation $X^{p}-X=P_{i}$ where $P_{i}$ is a polynomial in $\alpha_{0}, \ldots, \alpha_{i-1}, a_{0}, \ldots, a_{i}$ that all belong to the valuation ring of $K_{i-1}$. Thus, according to proposition 2.1, $K_{i} / K_{i-1}$ is unramified and so is $L=K_{n-1}$ over $K$.

We wish to complete these statements by considering all higher ramification groups for all Artin-Schreier-Witt extensions. Under the assumption that $\kappa$ is finite we shall achieve this goal.

Indeed, in the usual framework of local class field theory, i.e. when $K$ has finite residue field, the existence theorem (e.g. [14], Chap. $V, \S 4)$ is essentially the statement that the norm completion of $K^{*}$ and the completion with respect to its open subgroups of finite index are the same. Generalizing the reciprocity law for finite abelian extensions, it gives rise to an isomorphism of topological groups between this latter completion and the maximal abelian Galois group $G_{K}$ over $K$, that we shall denote by $\omega_{K}$. Moreover, for all integers $u \geq 0$, it induces isomorphisms of topological groups $U_{K}^{(u)} \stackrel{\simeq}{\longrightarrow} G_{K}^{(u)}$, where the subgroups $U_{K}^{(u)}$ are defined to be $1+\mathfrak{p}_{K}^{u}$ 
and form a basis of neighbourhoods of 1 in $U_{K}$. Therefore, in our setup, the existence theorem yields isomorphisms of topological groups:

$$
K^{*} / K^{* p^{n}} \stackrel{\cong}{\rightarrow} G_{p^{n}}
$$

and for all $u \geq 0$ :

$$
U_{K}^{(u)} K^{* p^{n}} / K^{* p^{n}} \stackrel{\simeq}{\rightrightarrows} G_{p^{n}}^{(u)}
$$

The idea is then to apply Pontryagin duality to the Artin-Schreier-Witt symbol so as to describe the filtration $\left\{U_{K}^{(u)} K^{* p^{n}} / K^{* p^{n}}\right\}_{u \geq 0}$ by orthogonality, and thus the ramification groups of $G_{p^{n}}$. Note that $W_{n}(K) / \wp\left(W_{n}(K)\right)$ is a discrete abelian group and $K^{*} / K^{* p^{n}}$ is an abelian profinite group that are both annihilated by $p^{n}$. Thus Pontryagin duality coincides with the $\operatorname{Hom}\left(-, W_{n}\left(\mathbb{F}_{p}\right)\right)$ duality on both of these groups since $W_{n}\left(\mathbb{F}_{p}\right)$ is canonically isomorphic to $\mathbb{Z} / p^{n} \mathbb{Z}$. Moreover, it establishes isomorphisms of topological groups between each group and its bidual. For further information about Pontryagin duality, we shall refer the reader to ([8], Chap.2, §9).

The crucial step is then to give an explicit formulation to compute the Artin-Schreier-Witt symbol. When $n=1$, this was already done by Schmid [10] :

Proposition 2.2 (Schmid Formula). If $a \in K$ and $b \in K^{*}$, then:

$$
[a, b)=\operatorname{Tr}_{\kappa / \mathbb{F}_{p}}\left(\operatorname{Res}\left(a \frac{d b}{b}\right)\right) .
$$

Here, the residue is defined as follows. If $f$ is in $K$, then $f d T$ is a differential form of $K$. The coefficient before $T^{-1}$ is called the residue of $f d T$ and denoted by $\operatorname{Res}(f d T)$. One may prove that it does not depend on the choice of the uniformizer.

We should stress the fact that the Schmid formula is essentially based on the more general identity $[a, b)=[c, t)$ where $c=\operatorname{Res}\left(a \frac{d b}{b}\right)$ for $n=1$. This remark will be of great use in the following (see lemma 3.1).

In the beautiful treatement of Serre ([13], Chap. XIV, §6), the Schmid formula is originally used to prove the existence theorem in characteristic $p$. Indeed, when the universal norm group $\mathcal{D}_{K}$ of $K$ is trivial the existence theorem yields a continuous injection from $K^{*}$ into $G_{K}$. In characteristic $p$, this is proposition 16 of ([13], Chap. XIV, §5) arising from the Schmid formula: 
Proposition 2.3. Let $K$ be a local field of characteristic $p$ with finite residue field. If an element $b \in K^{*}$ is a norm from every cyclic extension of $K$ of degree $p$, then $b \in K^{* p}$.

Therefore, $\mathcal{D}_{K}$ is divisible and thus $\mathcal{D}_{K}=\cap_{n} K^{* n}=1$. This proposition also proves that the kernel on the right in the Artin-Schreier-Witt symbol is trivial, whereas its kernel on the left was already clearly trivial under the Artin-Schreier-Witt theory.

Now, as an explicit computation for the Artin-Schreier-Witt symbol on $K / \wp(K) \times K^{*} / K^{* p}$, the Schmid formula allows us to express the ramification groups for the maximal abelian extension of exponent $p$ over $K$ in terms of subgroups of $H_{p}=\operatorname{Hom}\left(K / \wp(K), W_{1}\left(\mathbb{F}_{p}\right)\right)$. However, we delay the proof until later, since it will be included in the more general proof of theorem 1.1 in section 5 .

The consideration of the maximal abelian extensions of exponent $p^{n}$ over $K$ makes appeal to Witt vectors according to the classical Artin-SchreierWitt theory. As a set, $W(K)$ consists of infinite sequences $\left(x_{0}, x_{1}, \ldots\right)$ with components in $K$. This set is then functorially provided with two laws of operation by use of ghost components. If $\left(X_{0}, X_{1}, \ldots\right)$ is a sequence of indeterminates, we define the sequence of its ghost components $\left(X^{(0)}, X^{(1)}, \ldots\right)$ by:

$$
X^{(l)}=\sum_{i=0}^{l} p^{i} X_{i}^{p^{p-i}}=X_{0}^{p^{l}}+p X_{1}^{p^{l-1}}+\ldots+p^{l} X_{l} .
$$

If $R$ is an arbitrary ring, this gives rise to a map from $W(R)$ to $R^{\mathbb{N}}$ that we shall call the ghost map and denote by $\Gamma_{R}$. Now, the main difficulty stems from the following observation. When $R$ has characteristic 0 , the ghost map is injective and it is moreover bijective if $p$ is invertible in $R$. But in characteristic $p$, the ghost map is no longer injective. We thus provide $W(R)$ with two laws of operation that come from the usual laws of $R^{\mathbb{N}}$ under $\Gamma_{R}$ when $R$ has characteristic 0 . According to [17], these operations are given by a polynomial formula that we then use to define sum and product on $W(K)$.

We then define on $W(K)$ a shifting operator $V$ by :

$$
V:=\left(x_{0}, x_{1}, \ldots\right) \mapsto\left(0, x_{0}, x_{1}, \ldots\right) .
$$

In particular, for every $n \geq 1$, the ring $W_{n}(K)$ of truncated Witt vectors is the quotient ring $W(K) / V^{n} W(K)$ and one can pass from $W(K)$ to $W_{n}(K)$ via the truncation map $t_{n}$ defined as $\left(x_{0}, \ldots\right) \mapsto\left(x_{0}, \ldots, x_{n-1}\right)$. Then $W(K)$ 
is the inverse limit of all $W_{n}(K)$ with respect to the truncation maps and as such one can provide it with the $p$-adic topology. At last, let us mention the relation $V \circ F=F \circ V=\mathbf{p}$ where $F$ is the additive morphism $\left(x_{0}, \ldots\right) \mapsto$ $\left(x_{0}^{p}, \ldots\right)$ and where $\mathbf{p}$ is the multiplication by $p$; this implies in particular that $\wp$ and $V$ commute.

Since $W_{1}(K)$ is the additive group of $K$, we are led to define for Witt vectors of any length an explicit formulation of the Artin-Schreier-Witt symbol that extends the Schmid formula.

\section{The Schmid-Witt symbol}

For arbitrary $n \geq 1$, we shall develop a general formula to compute the Artin-Schreier-Witt symbol on $W_{n}(K) / \wp\left(W_{n}(K)\right) \times K^{*} / K^{* p^{n}}$. Witt [17] did it precisely for a $p$-adic field in the language of invariants of algebras. Drawing our inspiration from his formula, we shall prove it carefully in characteristic $p$.

The Artin-Schreier-Witt pairing is given by the theory of the same name:

$$
\begin{array}{cl}
W_{n}(K) / \wp\left(W_{n}(K)\right) \times K^{*} / K^{* p^{n}} & \rightarrow W_{n}\left(\mathbb{F}_{p}\right) \\
\left(a+\wp\left(W_{n}(K)\right), b . K^{* p^{n}}\right) & \mapsto[a, b):=(b, L / K)(\alpha)-\alpha,
\end{array}
$$

for some $\alpha=\left(\alpha_{0}, \ldots, \alpha_{n-1}\right) \in W_{n}\left(K^{\text {sep }}\right)$ such that $\wp(\alpha)=a$ and where $L=K\left(\alpha_{0}, \ldots, \alpha_{n-1}\right)$. Besides, $(b, L / K)$ is the reciprocity law of $b$ in $L / K$; it is also $\omega_{K}(b)$ restricted to $L$.

The notation $[., .)_{n}$ would be more convenient but for simplicity we shall rather use the notation [., .) even if it does not specify the index $n$. Anyway, we shall always fix an arbitrary integer $n \geq 1$ in what follows. Only section 2 dealt with the Artin-Schreier-Witt symbol for $n=1$, that is also called the Artin-Schreier symbol simply. Besides, note that the square bracket [ stands for the additivity of the group $W_{n}(K) / \wp\left(W_{n}(K)\right)$ whereas the bracket ) is related to the multiplicativity of $K^{*} / K^{* p^{n}}$.

This Artin-Schreier-Witt pairing satisfies the following:

Proposition 3.1. (i) The Artin-Schreier-Witt symbol is bilinear.

(ii) For every $a \in K,[a, b)=0$ if and only if $b$ is a norm in the extension $K\left(\alpha_{0}, \ldots, \alpha_{n-1}\right) / K$ where $\alpha=\left(\alpha_{0}, \ldots, \alpha_{n-1}\right)$ is such that $\wp(\alpha)=a$. (iii) For all $a \in K$ and $b \in K^{*},[V a, b)=V[a, b)$ where $V$ is the shifting operator.

Moreover, according to the existence theorem we claim: 
Proposition 3.2. The Artin-Schreier-Witt symbol is non-degenerate.

Proof. Let $a \in W_{n}(K)$ and suppose that $\omega_{K}(b)(\alpha)-\alpha=0$ for all $b$ 's in $K^{*}$. By the existence theorem, $\omega_{K}\left(K^{*}\right)$ is dense in $G_{K}$, thus $G_{K}$ fixes the extension $K(\alpha)$ defined by $a$, so $\alpha \in W_{n}(K)$ and $a \in \wp\left(W_{n}(K)\right)$.

Now let $b \in K^{*}$. If $\omega_{K}(b)(\alpha)-\alpha=0$ for all $a \in W_{n}(K)$ then $\omega_{K}(b)$ fixes all cyclic extensions of degree dividing $p^{n}$, thus it fixes all finite abelian extensions of exponent $p^{n}$ by taking the union and so it fixes the maximal abelian extension of exponent $p^{n}$ over $K$. Therefore, the image of $\omega_{K}(b)$ is the identity in $G_{p^{n}}$ which means that $b \in K^{* p^{n}}$ under the existence theorem. The converse is trivial.

The main idea of this section is to consider the Schmid formula in relation with the so-called ghost components of some Witt vector. For $a \in W_{n}(K)$ and $b \in K^{*}$, the idea is then to switch temporarily to characteristic 0 by lifting $a$ and $b$ to some elements $A$ and $B$ in $W_{n}(R((T)))$ and $R((T))^{*}$ respectively where $R$ is any complete discrete valuation ring of characteristic 0 with residue field $\kappa=\mathbb{F}_{q}$. The main observation is that the ring $R=W\left(\mathbb{F}_{q}\right)$ satisfies this property (see [13], Chap. II, $\S$, Thm.7). We then define an explicit pairing on $W_{n}(R((T))) \times R((T))^{*}$ with value in $W_{n}(R)$ : given $A$ and $B$ it returns a Witt vector $(A, B)$ in $W_{n}(R)$ given by its ghost components that are expressed as the residues of some elements in $R((T))$ generalizing in this way the Schmid formula. This is the "Residuenvektor" of [17]. This pairing is such that after reducing to $W_{n}(K) \times K^{*}$ we get a pairing that we shall call the Schmid-Witt symbol and that corresponds to the Artin-Schreier-Witt symbol. This is proposition 3.4. In proposition 3.5 we then give another formulation for the Schmid-Witt symbol that does not use the reduction to $W_{n}(K) \times K^{*}$. The present section is thus intended to provide a thorough exposition of the details of this process.

Note that $X_{0}=X^{(0)}$. Therefore, the ghost map is bijective from $W_{1}(A)$ onto $A$ whatever the characteristic of the ring $A$ is. This is the reason why the Schmid formula for $n=1$ does not use any lift at all, following up our reasoning.

Let $a \in W_{n}\left(\mathbb{F}_{q}((T))\right)$, write $a=\left(a_{i}\right)_{i}$ with $a_{i}=\sum_{v \geq v_{i}} a_{i, v} T^{i} \in \mathbb{F}_{q}((T))$ and $a_{i, v_{\imath}} \neq 0$. Let $b \in \mathbb{F}_{q}((T))^{*}$, we write $b=b_{m} T^{m}+$ h.o.t. with $b_{m} \in \mathbb{F}_{q}^{*}$ (the abbreviation h.o.t. stands for higher order terms). We shall lift $a$ and $b$ to $A \in W_{n}\left(W\left(\mathbb{F}_{q}((T))\right)\right)$ and $B \in W\left(\mathbb{F}_{q}\right)((T))^{*}$ respectively as follows:

$$
A=\left(A_{i}\right)_{i=0}^{n-1} \in W_{n}\left(W\left(\mathbb{F}_{q}\right)((T))\right),
$$


with, for all $i \in\{0, \ldots, n-1\}$ :

$$
A_{i}=\sum_{v \geq v_{i}} A_{i, v} T^{i} \text { where } A_{i, v} \in W\left(\mathbb{F}_{q}\right) \text { and }\left(A_{i, v}\right)_{0}=a_{i, v}
$$

and:

$$
B=\sum_{l \geq m} B_{l} T^{l} \in W\left(\mathbb{F}_{q}\right)((T))^{*}
$$

with $\left(B_{l}\right)_{0}=b_{l}$, for all $l \leq m$.

In particular, if we provide $W\left(\mathbb{F}_{q}\right)((T))$ with the usual valuation, then each $A_{i}$ has valuation $v_{K}\left(a_{i}\right)$ and $B$ has valuation $v_{K}(b)$.

Under the identification $K=\mathbb{F}_{q}((T))$, we then define a pairing $W_{n}(K) \times$ $K^{*} \rightarrow W_{n}\left(\mathbb{F}_{p}\right)$ given by the left vertical line in the following commutative diagram:

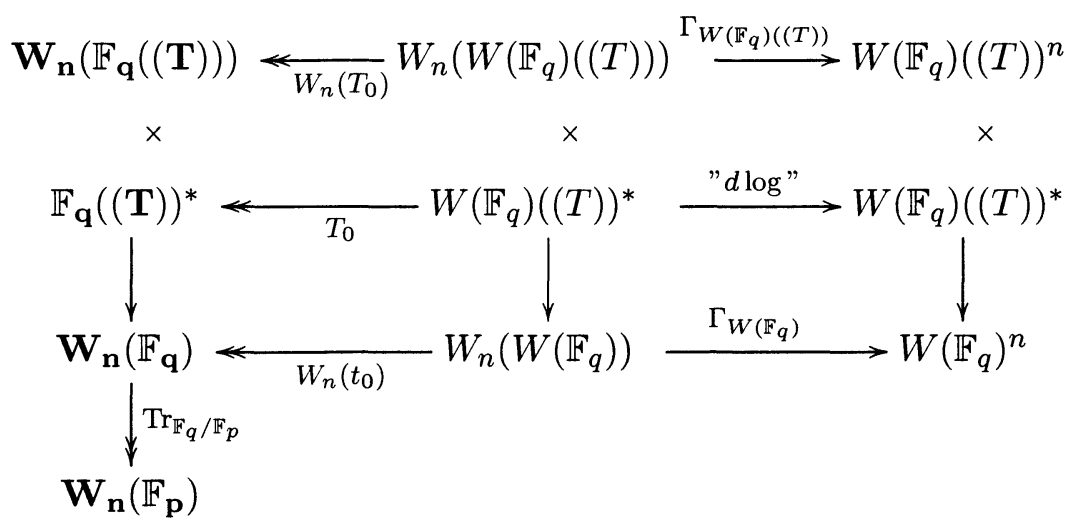

where the vertical and horizontal arrows are defined as follows:

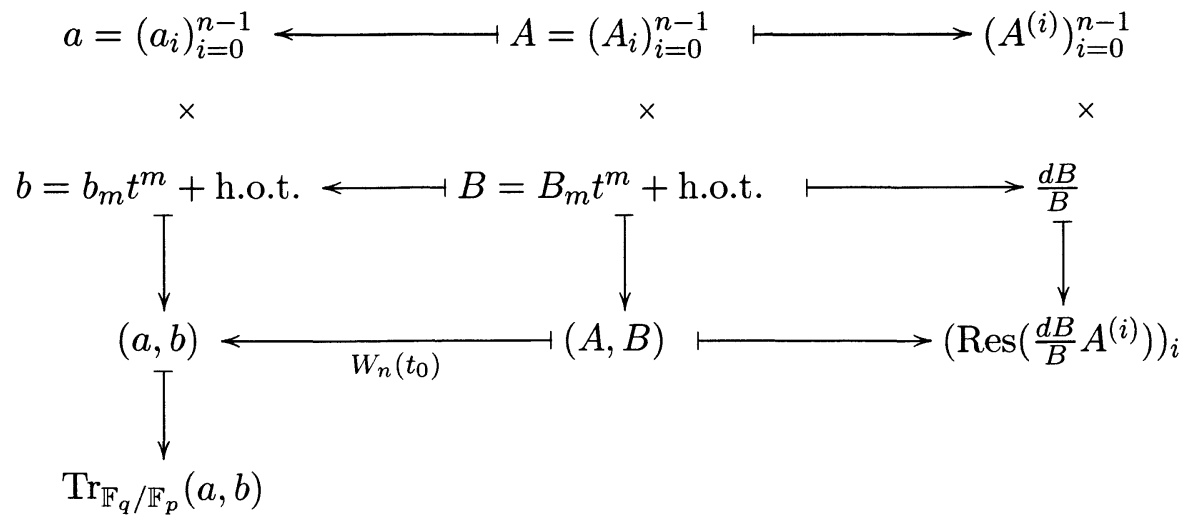


Here, the trace on $W_{n}\left(\mathbb{F}_{q}\right)$ is defined as follows:

$$
\forall x \in W_{n}\left(\mathbb{F}_{q}\right), \quad \operatorname{Tr}_{\mathbb{F}_{q} / \mathbb{F}_{p}}(x):=\sum_{i=0}^{d-1} F^{i} \cdot x,
$$

with respect to the Witt addition, where $d$ is such that $q=p^{d}$.

Note that the Witt vector $(A, B)$ defined by its ghost components should lie in $W_{n}\left(W\left(\mathbb{F}_{q}\right)\left[\frac{1}{p}\right]\right)$ a priori. However, according to Satz 4 of [17], each component of $(A, B)$ is a polynomial with integer coefficients in the indeterminates $B_{m}^{-1}, B_{l}$, and $A_{i, v}$ for all $l \geq m, i \geq 0$ and $v \geq v_{i}$. Moreover, $B_{m}^{-1}$ lies in $W\left(\mathbb{F}_{q}\right)$ since its first component $b_{m}$ is a unit in $\mathbb{F}_{q}$. Therefore, the vector $(A, B)$ does belong to $W_{n}\left(W\left(\mathbb{F}_{q}\right)\right)$ and the diagram is well defined. Furthermore, each component of $(a, b)$ is a polynomial with integer coefficients evaluated in $b_{m}^{-1}, b_{l}$ and $a_{i, v}$ since the first component of the sum (resp. the product) of two Witt vectors is the sum (resp. the product) of their first components.

Therefore, the Witt vector $(a, b) \in W_{n}\left(\mathbb{F}_{q}\right)$ is well defined and it does not depend on the choice of the lifts $A$ and $B$. We call it the Schmid-Witt symbol of $a$ and $b$.

Clearly, the Schmid-Witt symbol satisfies the following:

Proposition 3.3. (i) The Schmid-Witt pairing is bilinear.

(ii) For all $a \in W_{n}(K)$ and $b \in K^{*}:(V a, b)=V(a, b)$.

In order to show that the Artin-Schreier-Witt symbol and the SchmidWitt symbol are actually the same on $W_{n}(K) / \wp\left(W_{n}(K)\right) \times K^{*} / K^{* p^{n}}$, we first prove one crucial lemma:

Lemma 3.1. Let $a \in W_{n}(K)$ and $b \in K^{*}$. If $c=(a, b) \in W_{n}\left(\mathbb{F}_{q}\right)$, then:

$$
[a, b)=[c, T),
$$

where $[.,):. W_{n}(K) \times K^{*} \rightarrow W_{n}\left(\mathbb{F}_{p}\right)$ is the Artin-Schreier-Witt pairing.

Proof. The proof is mainly due to [17], we shall rewrite it to show how the computation of the Schmid-Witt symbol works. It is based on the following relation:

$$
x=\sum_{i=0}^{n-1} V^{i}\left\{x_{i}\right\}
$$

for all Witt vectors $x=\left(x_{0}, \ldots, x_{n-1}\right)$ in $W_{n}(K)$ where each $\left\{x_{i}\right\}$ is the Witt vector $\left(x_{i}, 0, \ldots, 0\right)$. 
We first assume that $b=T$ and we write $a_{0}=\sum_{v \in \mathbb{Z}} a_{0, v} T^{v}$ so that:

$$
a=\left\{a_{0,0}\right\}+\left\{\sum_{v>0} a_{0, v} T^{v}\right\}+\sum_{v>0}\left\{a_{0,-v} T^{-v}\right\}+\Omega a
$$

for some Witt vector $\Omega a$ in $W_{n}(K)$. Note that since the first component of a sum is the sum of the first components, $(\Omega a)_{0}=0$ and there exists some Witt vector $\omega=\left(\omega_{0}, \ldots, \omega_{n-1}\right) \in W_{n}(K)$ such that $\Omega a=V \omega$.

Besides, by bilinearity of the Artin-Schreier-Witt symbol, one gets:

$$
[a, T)=\left[\left\{a_{0,0}\right\}, T\right)+\left[\left\{\sum_{v>0} a_{0, v} T^{v}\right\}, T\right)+\sum_{v>0}\left[\left\{a_{0,-v} T^{-v}\right\}, T\right)+\Omega a .
$$

First, since $\left\{a_{0,0}\right\}$ lie in $W_{n}\left(\mathbb{F}_{q}\right)$, then $\left(\left\{a_{0,0}\right\}, T\right)=\left\{a_{0,0}\right\}$ for if $A$ is a lift for $\left\{a_{0,0}\right\}$ in the way we defined the Schmid-Witt symbol, then $A$ is in $W_{n}\left(W\left(\mathbb{F}_{q}\right)\right)$ so that all its ghost components $A^{(i)}$ are constant, thus $\operatorname{Res}\left(\frac{d B}{B} A^{(i)}\right)=A^{(i)}$ for each $i$, hence $(A, T)=A$ and so $\left(\left\{a_{0,0}\right\}, T\right)=\left\{a_{0,0}\right\}$. Thereby, $\left[\left(\left\{a_{0,0}\right\}, T\right), T\right)=\left[\left\{a_{0,0}\right\}, T\right)$.

Next, the Witt vector $a_{>0}=\left\{\sum_{v>0} a_{0, v} T^{v}\right\}$ clearly belongs to $\left(\mathfrak{p}_{K}, \ldots, \mathfrak{p}_{K}\right)$ and then it is in $\wp\left(W_{n}(K)\right)$ (we shall come back to this property in section 6 with proposition 6.1 since it is actually a more general statement when the residue field of $K$ is simply perfect). Thus $\left[a_{>0}, T\right)=0$. On the other hand, if $A$ is a lift for $a_{>0}$ with regards to the SchmidWitt symbol, then all ghost components of $A$ have strictly positive valuation, hence $\operatorname{Res}\left(\frac{d T}{T} A^{(i)}\right)=0$ for each $i$ and so $\left(a_{>0}, T\right)=0$. Therefore $\left[\left(a_{>0}, T\right), T\right)=\left[a_{>0}, T\right)$ again.

Then, according to lemma 3.2 that follows, each $\left\{a_{0,-v} T^{-v}\right\}$ for $v>0$ is such that $\left[\left\{a_{0,-v} T^{-v}\right\}, T\right)=0$. We write $a_{<0}$ for $\left\{a_{0,-v} T^{-v}\right\}$. Besides, when computing the Schmid-Witt symbol $\left(a_{<0}, T\right)$ one can lift $a_{<0}$ to some Witt vector $A$ of the type $\left\{A_{0,-v} T^{-v}\right\}$ with $A_{0,-v} \in W\left(\mathbb{F}_{q}\right)$ so that all $A^{(i)}$ are linear combinations of strictly negative powers of $T$ and as such satisfy $\operatorname{Res}\left(\frac{d T}{T} A^{(i)}\right)=0$ thus $\left(a_{<0}, T\right)=0$ and $\left[\left(a_{<0}, T\right), T\right)=\left[\left(a_{<0}, T\right)\right.$.

Therefore, by bilinearity of both the Artin-Schreier-Witt symbol and the Schmid-Witt symbol we get:

$$
[(a, T), T)-[a, T)=[(\Omega a, T), T)-[\Omega a, T) .
$$

Since $\Omega a=V \omega$ for some $\omega \in W_{n}(K)$, one may iterate the process and construct successively Witt vectors $\Omega^{i} a=V^{i} \omega_{i}$ with $\omega_{i} \in W_{n}(K)$ such that:

$$
\forall i \geq 0,[(a, T), T)-[a, T)=\left[\left(\Omega^{i} a, T\right), T\right)-\left[\Omega^{i} a, T\right) .
$$

In particular, for $i=n$ we get $\Omega^{n} a=V^{n} \omega_{n}=0$ and thus $[(a, T), T)-$ $[a, T)=0$, as was to be shown. 
Finally, if $b=\epsilon T^{m}$ where $\epsilon$ is a unit in $K$ and $m>0$, then $T^{\prime}:=b T^{1-m}$ is a uniformizing element in $K$, whence:

$$
\begin{aligned}
{[a, b)=\left[a, T^{\prime} T^{m-1}\right) } & =(m-1)[a, T)+\left[a, T^{\prime}\right) \\
& =(m-1)[(a, T), T)+\left[\left(a, T^{\prime}\right), T^{\prime}\right)
\end{aligned}
$$

and we conclude by bilinearity of the Schmid-Witt symbol from what precedes, indeed:

$$
\begin{aligned}
{[(a, b), T) } & =(m-1)[(a, T), T)+\left[\left(a, T^{\prime}\right), T\right) \\
& =(m-1)[(a, T), T)+\left[\left(a, T^{\prime}\right), T^{\prime}\right)-\left[\left(a, T^{\prime}\right), b T^{-m}\right),
\end{aligned}
$$

where $b T^{-m}$ is a unit in $K$. Now $\left(a, T^{\prime}\right)$ lies in $W_{n}\left(\mathbb{F}_{q}\right)$ thus in $W_{n}\left(O_{K}\right)$ and according to corollary 2.1 this means that the corresponding Artin-SchreierWitt extension is unramified. Hence $\left[\left(a, T^{\prime}\right), b T^{-m}\right)=0$ since $b T^{-m}$ is a unit and so $[(a, b), T)=[a, b)$ as was to be shown.

In the above proof, we made use of the following statement due to Teichmüller in [15]:

Lemma 3.2. Let $a_{0} \in K^{*}$ and let $a=\left\{a_{0}\right\}$ be a Witt vector of length $n$ such that $a_{0}=\sum_{v>0} a_{0, v} T^{-v}$, i.e. $a_{0}$ is the linear combination of strictly negative powers of $T$. Then $[a, T)=0$.

Generalizing the Schmid formula, we finally get:

Proposition 3.4 (Schmid-Witt Formula). If $a \in W_{n}(K)$ and if $b \in K^{*}$, then:

$$
[a, b)=\operatorname{Tr}_{\mathbb{F}_{q} / \mathbb{F}_{p}}((a, b)) .
$$

Proof. According to lemma 3.1, one may take $b=T$ and suppose that $a$ is a constant, i.e. an element in $W_{n}\left(\mathbb{F}_{q}\right)$ where $\mathbb{F}_{q}=\kappa$. Then, proposition 3.4 amounts to the same as saying:

$$
[a, T)=\operatorname{Tr}_{\mathbb{F}_{q} / \mathbb{F}_{p}}(a),
$$

since $(a, T)=a$ when $a$ is constant.

So let $\alpha \in W_{n}\left(\kappa^{\mathrm{sep}}\right)$ be a root of the equation $\wp(\alpha)=a$, and let $\kappa^{\prime} / \kappa$ be the corresponding cyclic extension of degree $p^{k} \leq p^{n}$ under Artin-SchreierWitt theory. We set $K^{\prime}:=\kappa^{\prime}((T))$ : it is an unramified extension of $K$. Thus, according to ([13], Chap. XIII, $\S 4$, prop. 13) one gets: $\left(T, K^{\prime} / K\right)=$ $F_{q}$ where $F_{q}$ denotes the canonical generator of $\operatorname{Gal}\left(K^{\prime} / K\right) \simeq \operatorname{Gal}\left(\kappa^{\prime} / \kappa\right)$ defined by $x \mapsto x^{q}$ with $q=|\kappa|$. Therefore, writing $q=p^{l}$ and since $F_{q}$ 
commutes with $\wp$, it comes:

$$
\begin{aligned}
{[a, T] } & =F_{q} \alpha-\alpha \\
& =\left(\alpha_{0}^{q}, \ldots, \alpha_{n-1}^{q}\right)-\left(\alpha_{0}, \ldots, \alpha_{n-1}\right) \\
& =\left(\alpha_{0}^{p^{l}}, \ldots\right)-\left(\alpha_{0}^{p^{l-1}}, \ldots\right)+\left(\alpha_{0}^{p^{l-1}}, \ldots\right)+\ldots+\left(\alpha_{0}^{p}, \ldots\right)-\left(\alpha_{0}, \ldots\right) \\
& =\wp\left(\alpha_{0}^{p^{l-1}}, \ldots\right)+\ldots+\wp\left(\alpha_{0}, \ldots\right) \\
& =\wp\left(F_{q}^{l-1}(\alpha)\right)+\wp\left(F_{q}^{l-2}(\alpha)\right)+\ldots+\wp(\alpha) \\
& =F_{q}^{l-1}(a)+\ldots+F_{q}(a)+a \\
& =\operatorname{Tr}_{\mathbb{F}_{q} / \mathbb{F}_{p}}(a)
\end{aligned}
$$

for $\mathbb{F}_{q}$ is cyclic of order $l$ over $\mathbb{F}_{p}$.

Note that Teichmüller's lemma 3.2 is true when the residue field of $K$ is perfect whereas the Schmid-Witt formula is valid only when the residue field is finite. It implies in particular that the Schmid-Witt symbol is nondegenerate in the usual framework of local class field theory.

We conclude this section with an equivalent construction for the SchmidWitt symbol corresponding to the idea that the $(n-1)$ th ghost component of a Witt vector contains all the needed information. This will make its computation easier:

Proposition 3.5. For every $a \in W_{n}(K)$ and every $b \in K^{*}$, if $A$ and $B$ are lifts of $a$ and $b$ respectively with regards to the Schmid-Witt symbol, then:

$$
\operatorname{Tr}_{\mathbb{F}_{q} / \mathbb{F}_{p}}(a, b)=t_{n}\left(\operatorname{Tr}_{\mathbb{F}_{q} / \mathbb{F}_{p}} \operatorname{Res}\left(\frac{d B}{B} A^{(n-1)}\right)\right),
$$

where $t_{n}: W\left(\mathbb{F}_{p}\right) \rightarrow W_{n}\left(\mathbb{F}_{p}\right)$ is the truncation map.

Proof. The proof is based on the following observation. If $Y=\left(Y_{0}, \ldots, Y_{n-1}\right)$ is a Witt vector in $W_{n}\left(\mathbb{F}_{q}\right)$, then, for every integer $k \geq 1$ we have:

$$
Y^{p^{k}}=\left\{Y_{0}^{p^{k}}\right\}+p^{k+1} Z
$$

for some Witt vector $Z \in W_{n}\left(\mathbb{F}_{q}\right)$. This is particularly due to the relation $V \circ F=F \circ V=\mathbf{p}$.

So, let $X=\left(X_{i}\right)_{i}$ with $X_{i} \in W\left(\mathbb{F}_{q}\right)$ denoting the Witt vector $(A, B)$ and let $x=\left(x_{i}\right)_{i}$ stand for $(a, b)$. Recall that these are related by $\left(X_{i}\right)_{0}=x_{i}$ for all $i$. We are thus going to prove that $\operatorname{Tr}_{\mathbb{F}_{q} / \mathbb{F}_{p}} x=t_{n}\left(\operatorname{Tr}_{\mathbb{F}_{q} / \mathbb{F}_{p}} X^{(n-1)}\right)$. According to the previous remark, there exist Witt vectors $Z_{i}$ in $W_{n}\left(\mathbb{F}_{q}\right)$ 
such that:

$$
\begin{aligned}
\operatorname{Tr}_{\mathbb{F}_{q} / \mathbb{F}_{p}} X^{(n-1)} & =\operatorname{Tr}_{\mathbb{F}_{q} / \mathbb{F}_{p}} \sum_{i=0}^{n-1} p^{i} X_{i}^{p^{n-1-\imath}} \\
& =\sum_{i=0}^{n-1} p^{i} \operatorname{Tr}_{\mathbb{F}_{q} / \mathbb{F}_{p}}\left(X_{i}^{p^{n-1-i}}\right) \\
& =\sum_{i=0}^{n-1} p^{i} \operatorname{Tr}_{\mathbb{F}_{q} / \mathbb{F}_{p}}\left(\left\{\left(X_{i}\right)_{0}^{p^{n-1-i}}\right\}+p^{n-i} Z_{i}\right) .
\end{aligned}
$$

Now, since $F$ is the identity map on $W_{n}\left(\mathbb{F}_{p}\right), p^{i}=V^{i}$ on $W_{n}\left(\mathbb{F}_{p}\right)$ and by linearity of the trace we get:

$$
\begin{aligned}
\operatorname{Tr}_{\mathbb{F}_{q} / \mathbb{F}_{p}} X^{(n-1)} & =\sum_{i=0}^{n-1} V^{i} \operatorname{Tr}_{\mathbb{F}_{q} / \mathbb{F}_{p}}\left(\left\{\left(X_{i}\right)_{0}^{p^{n-1-\imath}}\right\}\right)+V^{n} \sum_{i=0}^{n-1} \operatorname{Tr}_{\mathbb{F}_{q} / \mathbb{F}_{p}}\left(Z_{i}\right) \\
& =\sum_{i=0}^{n-1} V^{i} \operatorname{Tr}_{\mathbb{F}_{q} / \mathbb{F}_{p}}\left(\left\{\left(X_{i}\right)_{0}^{p^{n-1-i}}\right\}\right) \bmod V^{n} W\left(\mathbb{F}_{q}\right) .
\end{aligned}
$$

Then, once we observe that $\operatorname{Tr}_{\mathbb{F}_{q} / \mathbb{F}_{p}}\left(\left\{w^{p^{k}}\right\}\right)=\operatorname{Tr}_{\mathbb{F}_{q} / \mathbb{F}_{p}}(\{w\})$ for each $w \in \mathbb{F}_{q}$ and for all integers $k \geq 1$ and that the trace commutes with $V$, we finally get:

$$
\operatorname{Tr}_{\mathbb{F}_{q} / \mathbb{F}_{p}} X^{(n-1)}=\operatorname{Tr}_{\mathbb{F}_{q} / \mathbb{F}_{p}} \sum_{i=0}^{n-1} V^{i}\left\{\left(X_{i}\right)_{0}\right\} \quad \bmod V^{n} W\left(\mathbb{F}_{q}\right),
$$

which means that $t_{n}\left(\operatorname{Tr}_{\mathbb{F}_{q} / \mathbb{F}_{p}} X^{(n-1)}\right)=\operatorname{Tr}_{\mathbb{F}_{q} / \mathbb{F}_{p}} x$ as was to be shown.

Likewise, we get a formula in characteristic $p$ similar to that of Satz 18 in [17] for Witt vectors of length $n$ over a $p$-adic field. Note that Schmid [12] does not use this formula even though it makes the computation of the Artin-Schreier-Witt symbol simpler.

\section{The reduced form of a Witt vector}

One way to make the computation of the Artin-Schreier-Witt symbol even easier is to prove that on the local field $K$ every Witt vector of length $n$ is congruent modulo $\wp\left(W_{n}(K)\right)$ to a Witt vector with suitable components. This reduction was already mentioned by Schmid in [12]:

Proposition 4.1. Let $a=\left(a_{0}, \ldots, a_{n-1}\right)$ be a Witt vector in $W_{n}(K)$. Then $a$ is congruent modulo $\wp\left(W_{n}(K)\right)$ to a Witt vector $a^{\prime}=\left(a_{0}^{\prime}, \ldots, a_{n-1}^{\prime}\right)$ such 
that for each index $i$, either the component $a_{i}^{\prime}$ lies in $O_{K}$ or its valuation $v_{K}\left(a_{i}^{\prime}\right)$ is negative and not divisible by $p$.

The Witt vector $a^{\prime}$ is said to be reduced and it is called the reduced form of $a$.

We then introduce the following:

Definition. If $a=\left(a_{0}, \ldots, a_{n-1}\right) \in W_{n}(K)$, one defines $M_{n}(a)$ to be:

$$
M_{n}(a):=\max _{i}\left\{-p^{n-1-i} v_{K}\left(a_{i}\right)\right\} \text {. }
$$

Since $v_{K}(0)=+\infty$, the value of $M_{n}(a)$ is either an integer or $+\infty$. Now, it is always an integer once we choose a non-zero Witt vector $a$. On $W_{n}(K)$ the $M_{n}$ function enjoys the following properties:

Proposition 4.2. For any Witt vectors $x$ and $y$ in $W_{n}(K)$, we have:

(1) Let $u \geq 0$. If $x \in W_{n}^{(u)} \backslash W_{n}^{(u-1)}$, then $M_{n}(x)=u$.

(2) If $x$ is reduced and if $M_{n}(x) \geq 1$, then $M_{n}(x)=-p^{n-j-1} v_{K}\left(x_{j}\right)$ for a unique $j$ in $\{0, \ldots, n-1\}$.

(3) Let $x$ and $y$ be two Witt vectors in $W_{n}(K)$, then $M_{n}(x+y) \leq$ $\max \left\{M_{n}(x), M_{n}(y)\right\}$.

(4) If $x \in W_{n}(K)$ and if $c \in W_{n}\left(\mathbb{F}_{p}\right)$, then $M_{n}(c x) \leq M_{n}(x)$

(5) If $c \in W_{n}\left(\mathbb{F}_{p}\right)$ is a unit, then $M_{n}(c x)=M_{n}(x)$. In particular: $M_{n}(-x)=M_{n}(x)$.

(6) If $M_{n}(x) \neq M_{n}(y)$, the equality $M_{n}(x+y)=\max \left(M_{n}(x), M_{n}(y)\right)$ holds.

(7) Either $M_{n}(\wp(x)) \leq 0$ or $p$ divides $M_{n}(\wp(x))$.

(8) If $x$ is reduced and if $h \in W_{n}(K)$, then either $M_{n}(x) \leq 0$ or $M_{n}(x) \leq$ $M_{n}(x+\wp(h))$.

We refer the reader to Chapter 4, Paragraph 4.3 of [16] for a detailed proof of these assertions.

Note that all these statements related to reduced Witt vectors are valid even when the residue field of $K$ is perfect. As a consequence of all these properties, one should mention:

Corollary 4.1. Let $u \geq 1$. If $x$ is a reduced Witt vector such that its image modulo $\wp\left(W_{n}(K)\right)$ lies in:

$$
\left(W_{n}^{(u)}+\wp\left(W_{n}(K)\right)\right) / \wp\left(W_{n}(K)\right) \backslash\left(W_{n}^{(u-1)}+\wp\left(W_{n}(K)\right)\right) / \wp\left(W_{n}(K)\right),
$$

then $x$ is in $W_{n}^{(u)}(K) \backslash W_{n}^{(u-1)}(K)$.

Proof. For simplicity, write $A^{(u)}:=\left(W_{n}^{(u)}+\wp\left(W_{n}(K)\right)\right) / \wp\left(W_{n}(K)\right)$ and denote by $\bar{x}$ the image of $x$ modulo $\wp\left(W_{n}(K)\right)$. Since $\bar{x} \notin A^{(u-1)}$ then $x \notin W_{n}^{(u-1)}$. Thus, by proposition $4.2, M_{n}(x) \geq u$. 
Now, $\bar{x} \in A^{(u)}$, hence there exist $z \in W_{n}^{(u)}(K)$ and $h \in W_{n}(K)$ such that $x=z+\wp(h)$, i.e. $z=x+\wp(-h)$. Thus, on the one hand, $M_{n}(z) \leq u$. On the other hand, according to proposition 4.2 again, we have: $M_{n}(x) \leq$ $M_{n}(x+\wp(-h))=M_{n}(z)$. Thereby, $M_{n}(x)=u$ and so $x \in W_{n}^{(u)}(K)$, as was to be shown.

The computation of the Schmid-Witt symbol for reduced Witt vectors gives:

Proposition 4.3. Let $0 \leq m \leq u$ be two positive integers.

Let $a \in W_{n}^{(m)}(K) \backslash W_{n}^{(m-1)}(K)$ be a reduced Witt vector of length $n$. We write $a=\left(a_{0}, \ldots, a_{n-1}\right)$ with $a_{i}=\sum_{v \geq v_{i}} a_{i, v} T^{v}$ and $a_{i, v_{i}} \in \kappa^{*}$, for each $i \geq 0$.

Let $b \in K^{*}$ such that its image modulo $K^{* p^{n}}$ is in $U_{K}^{(u)} K^{* p^{n}} / K^{* p^{n}}$ but not in $U_{K}^{(u-1)} K^{* p^{n}} / K^{* p^{n}}$. One may write $b=1+b_{u} T^{u}+$ h.o.t. with $b_{u} \in \kappa^{*}$. Then:

$$
\operatorname{Tr}_{\mathbb{F}_{q} / \mathbb{F}_{p}}(a, b)= \begin{cases}\left(0, \ldots, 0, \operatorname{Tr}_{\mathbb{F}_{q} / \mathbb{F}_{p}}\left(\left(-v_{j}\right) b_{u} a_{j, v_{j}}^{p^{n-1-j}}\right)\right) & \text { if } u=m \\ 0 & \text { if } u>m\end{cases}
$$

where $j$ is the only index such that $M_{n}(a)=-p^{n-1-j} v_{j}=m$ and $v_{j}=$ $v_{K}\left(a_{j}\right)$.

Proof. Recall that the field $K$ is identified with $\mathbb{F}_{q}((T))$.

Let $A \in W_{n}\left(W\left(\mathbb{F}_{q}\right)((T))\right)$ and $B \in W\left(\mathbb{F}_{q}\right)((T))^{*}$ be two lifts of $a$ and $b$ respectively with regards to the computation of the Schmid-Witt symbol as in section 3.2. According to proposition 3.5, we have:

$$
\operatorname{Tr}_{\mathbb{F}_{q} / \mathbb{F}_{p}}(a, b)=t_{n}\left(\operatorname{Tr}_{\mathbb{F}_{q} / \mathbb{F}_{p}}\left(\operatorname{Res}\left(\frac{d B}{B} A^{(n-1)}\right)\right) .\right.
$$

Let $v$ denote the usual valuation on $W\left(\mathbb{F}_{q}\right)((T))$. Since $W\left(\mathbb{F}_{q}\right)((T))$ is of characteristic 0 , then $A^{(n-1)}=\sum_{i \geq v_{n-1}} p^{i} A_{i}^{p^{n-1-i}}$ and since $a$ is reduced we get: $v\left(A^{(n-1)}\right)=\min _{i}\left\{p^{n-1-i} v_{i}\right\}=p^{n-1-j} v_{j}=-m$ for $v_{i}=v_{K}\left(a_{i}\right)=$ $v\left(A_{i}\right)$.

Clearly, one gets:

$$
\frac{d B}{B} A^{(n-1)}=u B_{u} p^{j} A_{j, v_{j}}^{p^{n-1-j}} T^{u-m-1}+\text { h.o.t. }
$$

In particular, if $u>m$ then $\operatorname{Res}\left(\frac{d B}{B} A^{(n-1)}\right)=0$ and so $\operatorname{Tr}_{\mathbb{F}_{q} / \mathbb{F}_{p}}(a, b)=0$. But if $u=m$, i.e. $u=-p^{n-1-j} v_{j}$, then:

$$
\operatorname{Res}\left(\frac{d B}{B} A^{(n-1)}\right)=\left(-v_{j}\right) p^{n-1} B_{u} A_{j, v_{\jmath}}^{p^{n-1-\jmath}},
$$


with $v_{j}$ prime to $p$. Thereby, when taking the trace of $\mathbb{F}_{q}$ over $\mathbb{F}_{p}$ it comes:

$$
\operatorname{Tr}_{\mathbb{F}_{q} / \mathbb{F}_{p}}\left(\operatorname{Res}\left(\frac{d B}{B} A^{(n-1)}\right)\right)=p^{n-1} \operatorname{Tr}_{\mathbb{F}_{q} / \mathbb{F}_{p}}\left(\left(-v_{j}\right) B_{u} A_{j, v_{j}}^{p^{n-1-j}}\right)
$$

since the trace is linear. Now recall that $\mathbf{p}^{n-1}=V^{n-1}$ on $W_{n}\left(\mathbb{F}_{p}\right)$, so that:

$$
\begin{aligned}
\operatorname{Tr}_{\mathbb{F}_{q} / \mathbb{F}_{p}}\left(\operatorname{Res}\left(\frac{d B}{B} A^{(n-1)}\right)\right) & =V^{n-1}\left(\operatorname{Tr}_{\mathbb{F}_{q} / \mathbb{F}_{p}}\left(\left(-v_{j}\right) B_{u} A_{j, v_{j}}^{p^{n-1-j}}\right)\right) \\
& =V^{n-1}\left(\operatorname{Tr}_{\mathbb{F}_{q} / \mathbb{F}_{p}}\left(\left(-v_{j}\right) b_{u} a_{j, v_{j}}^{p^{n-1-j}}\right), *, * \ldots\right),
\end{aligned}
$$

for $\operatorname{Tr}_{\mathbb{F}_{q} / \mathbb{F}_{p}}\left(x_{0}, x_{1}, \ldots\right)=\left(\operatorname{Tr}_{\mathbb{F}_{q} / \mathbb{F}_{p}}\left(x_{0}\right), \ldots\right)$ and $\left(x_{0}, x_{1}, \ldots\right)^{p^{k}}=\left(x_{0}^{p^{k}}, \ldots\right)$.

Thereby:

$$
\operatorname{Tr}_{\mathbb{F}_{q} / \mathbb{F}_{p}}(a, b)=\left(0, \ldots, 0, \operatorname{Tr}_{\mathbb{F}_{q} / \mathbb{F}_{p}}\left(\left(-v_{j}\right) b_{u} a_{j, v_{j}}^{p^{n-1-j}}\right),\right.
$$

as was to be shown.

\section{Proof of theorem 1.1}

We first check that the Artin-Schreier-Witt symbol induces an isomorphism between $G_{p^{n}}$ and $H_{p^{n}}$ that coincides with the Artin-Schreier-Witt isomorphism $\mathfrak{a s}_{n}$ under Pontryagin duality:

Proposition 5.1. The Artin-Schreier-Witt symbol gives rise to an isomorphism of topological groups:

$$
\psi_{p^{n}}: K^{*} / K^{* p^{n}} \stackrel{\simeq}{\longrightarrow} \operatorname{Hom}\left(W_{n}(K) / \wp\left(W_{n}(K)\right), W_{n}\left(\mathbb{F}_{p}\right)\right)
$$

given by $\left(b . K^{* p^{n}}\right) \mapsto\left\{\left(a+\wp\left(W_{n}(K)\right)\right) \mapsto[a, b)\right\}$.

In particular, under the existence theorem, it induces an isomorphism of topological groups:

$$
\psi_{p^{n}} \circ \omega_{K}^{-1}: G_{p^{n}} \stackrel{\simeq}{\longrightarrow} \operatorname{Hom}\left(W_{n}(K) / \wp\left(W_{n}(K)\right), W_{n}\left(\mathbb{F}_{p}\right)\right)
$$

that is identically equal to the Artin-Schreier-Witt isomorphism $\mathfrak{a s}_{n}$.

Proof. Since the Artin-Schreier-Witt symbol is non-degenerate, it induces injective group homomorphisms given by:

$$
\begin{array}{rlrr}
f: & K^{*} / K^{* p^{n}} & \hookrightarrow & \operatorname{Hom}\left(W_{n}(K) / \wp\left(W_{n}(K)\right), W_{n}\left(\mathbb{F}_{p}\right)\right) \\
b . K^{* p^{n}} & \mapsto & \left\{f_{b}: a+\wp\left(W_{n}(K)\right) \mapsto[a, b)\right\}
\end{array}
$$

and

$$
\begin{array}{llll}
g: & W_{n}(K) / \wp\left(W_{n}(K)\right) & \hookrightarrow & \operatorname{Hom}\left(K^{*} / K^{* p^{n}}, W_{n}\left(\mathbb{F}_{p}\right)\right) \\
& a+\wp\left(W_{n}(K)\right) & \mapsto & \left\{g_{a}: b \cdot K^{* p^{n}} \mapsto[a, b)\right\} .
\end{array}
$$


Now, $W_{n}(K) / \wp\left(W_{n}(K)\right)$ is discrete, thus the injection $g$ is continuous and its transposed map yields a continuous surjection denoted by $g^{\wedge}$ :

$$
\begin{aligned}
\operatorname{Hom}\left(\operatorname{Hom}\left(K^{*} / K^{* p^{n}}, W_{n}\left(\mathbb{F}_{p}\right)\right), W_{n}\left(\mathbb{F}_{p}\right)\right) \stackrel{g^{\wedge}}{\rightarrow} \\
\operatorname{Hom}\left(W_{n}(K) / \wp\left(W_{n}(K)\right), W_{n}\left(\mathbb{F}_{p}\right)\right)
\end{aligned}
$$

that is given by $g^{\wedge}(\chi): a+\wp\left(W_{n}(K)\right) \mapsto \chi \circ g\left(a+\wp\left(W_{n}(K)\right)\right)$.

Then, by Pontryagin duality, we get a continuous surjective homomorphism:

$$
\begin{array}{ccc}
K^{*} / K^{* p^{n}} & \rightarrow & \operatorname{Hom}\left(W_{n}(K) / \wp\left(W_{n}(K)\right), W_{n}\left(\mathbb{F}_{p}\right)\right) \\
b . K^{* p^{n}} & \mapsto & \left\{a+\wp\left(W_{n}(K)\right) \mapsto[a, b)\right\}
\end{array}
$$

and this latter is identically the map $f$ defined above. Hence $f$ is a continuous isomorphism. Since $K^{*} / K^{* p^{n}}$ and $\operatorname{Hom}\left(W_{n}(K) / \wp\left(W_{n}(K)\right), W_{n}\left(\mathbb{F}_{p}\right)\right)$ are compact, $f$ is an isomorphism of topological groups. We shall denote it by $\psi_{p^{n}}$.

Thereby, the composed map $\omega_{K}^{-1} \circ \psi_{p^{n}}$ yields an isomorphism of topological groups:

$$
G_{p^{n}} \stackrel{\simeq}{\longrightarrow} \operatorname{Hom}\left(W_{n}(K) / \wp\left(W_{n}(K)\right), W_{n}\left(\mathbb{F}_{p}\right)\right)
$$

given by: $\sigma \mapsto\left\{a+\wp\left(W_{n}(K)\right) \mapsto[a, b)\right\}$ where $b=\omega_{K}^{-1}(\sigma) \in K^{*}$. This isomorphism is the Artin-Schreier-Witt isomorphism $\mathfrak{a s}_{n}$ since $[a, b)=$ $\omega_{K}(b)(\alpha)-\alpha=\sigma(\alpha)-\alpha$ where $\wp(\alpha)=a$.

We then shall consider the ramification groups of $G_{p^{n}}$. Under the existence theorem, an integer $t \geq-1$ is called a jump in the filtration $\left\{U_{K}^{(u)} K^{* p^{n}} / K^{* p^{n}}\right\}_{u}$ of $U_{K} K^{* p^{n}} / K^{* p^{n}}$ if $U_{K}^{(t)} \neq U_{K}^{(t+1)} \bmod K^{* p^{n}}$. Now, $\kappa$ is perfect. Thus the filtration of $U_{K} K^{* p^{n}} / K^{* p^{n}}$ contains no jump greater or equal to 0 that is divisible by $p^{n}$ because $\kappa \subset \kappa^{p^{n}}$; thus, the same holds for the filtration of ramification groups in $G_{p^{n}}$.

We shall say that an integer $t \geq-1$ is a jump in the filtration $\left\{H_{p^{n}}^{(u)}\right\}_{u}$ if $H_{p^{n}}^{(u)} \neq H_{p^{n}}^{(u-1)}$. Note the existence of a shift by 1 with the definition of jumps for $G_{p^{n}}$. According to corollary 4.1, one may prove that no jump greater than 1 is divisible by $p^{n}$ in the filtration of $H_{p^{n}}$.

For each integer $u \geq 0$, we then define:

$$
\mathcal{S}_{p^{n}}^{(u)}:=\left\{b . K^{* p^{n}} \in K^{*} / K^{* p^{n}}:[a, b)=0, \forall a \in W_{n}^{(u)}\right\},
$$

i.e. $\mathcal{S}_{p^{n}}^{(u)}$ is the orthogonal group of $W_{n}^{(u)}(K)$ modulo $\wp\left(W_{n}(K)\right)$ in $K^{*} / K^{* p}$ with respect to the Artin-Schreier-Witt symbol. In particular, for $u=0$, it is the orthogonal of $\left(W_{n}\left(O_{K}\right) / \wp\left(W_{n}\left(O_{K}\right)\right)\right)$. Clearly, the groups $\mathcal{S}_{p^{n}}^{(u)}$, s 
form a decreasing filtration in $K^{*} / K^{*} p^{n}$. Then as a consequence of proposition 4.3, we state:

Proposition 5.2. For each integer $u \geq 1$, we get:

$$
\mathcal{S}_{p^{n}}^{(u-1)}=\left(U_{K}^{(u)} K^{* p^{n}}\right) / K^{* p^{n}}
$$

Proof. From proposition 4.3 and corollary $4.1,\left(U_{K}^{(u)} K^{* p^{n}}\right) / K^{* p^{n}} \subset \mathcal{S}_{p^{n}}^{(u-1)}$ if $u$ is not divisible by $p^{n}$.

Conversely, let $b \in K^{*}$ such that $\bar{b}$ does not lie in $\left(U_{K}^{(u)} K^{* p^{n}}\right) / K^{* p^{n}}$ and let us prove that it does not lie in $\mathcal{S}_{p^{n}}^{(u-1)}$ either. Let $j \leq u-1$ be the smallest integer such that $\bar{b} \in\left(U_{K}^{(j)} K^{* p^{n}}\right) / K^{* p^{n}}$. In particular $j$ is not divisible by $p^{n}$ according to a previous remark, thus $j=p^{k} v$ with $0 \leq k \leq n-1$ and $v \geq 1$ is prime to $p$. Besides, we may write: $b=$ $1+b_{j} T^{j}+$ h.o.t. with $b_{j} \in \mathbb{F}_{q}^{*}$. Then, by the non-zero functoriality of the trace, there exists $\gamma \in \mathbb{F}_{q}^{*}$ such that $\operatorname{tr}_{\mathbb{F}_{q} / \mathbb{F}_{p}}\left(\gamma v b_{j}\right) \neq 0$. Now, $\gamma=\alpha^{p^{n-1-j}}$ for some $\alpha \in \mathbb{F}_{q}^{*}$. Thus, if we consider the Witt vector $a=\left(a_{i}\right)_{i}$ defined by $a_{j}=\alpha T^{-v}+$ h.o.t and $a_{i}=0$ for all $i \neq j$, then $a$ is reduced and lies in $W_{n}^{(v)}$ thus in $W_{n}^{(u-1)}$ since $v \leq j \leq u-1$. Furthermore, according to theorem 3.4 and proposition $4.3,[a, b) \neq 0$. Thereby, $\bar{b}$ does not belong to $\mathcal{S}_{p^{n}}^{(u-1)}$, which proves that $\mathcal{S}_{p^{n}}^{(u-1)}=U_{K}^{(u)} K^{* p^{n}} / K^{* p^{n}}$ for every integer $u \geq 1$ that is not divisible by $p^{n}$.

If $u \geq 1$ is divisible by $p^{n}$, then $u$ is a jump neither for $H_{p^{n}}$ nor for $U_{K} K^{*} p^{n} / K^{*} p^{n}$, which completes the proof.

Whence the proof of theorem 1.1: for $u \geq 1$, it is just a direct consequence of proposition 5.2 and proposition 5.1. For $u=0$, it is exactly the statement that $G_{p^{n}}^{(0)}=G_{p^{n}}^{(1)}$ since the extension is wildly ramified.

A first consequence of theorem 1.1 is corollary 1.1 that we stated in the introduction. The proof essentially consists in writing out the inverse limit on all $G_{p^{n}}^{(u)}$ 's for $n \geq 1$ and $u \geq-1$. In particular, for $u=0, G_{p^{\infty}}^{(0)}$ is isomorphic to $\left\{\varphi \in H_{p^{\infty}}: \varphi\left(W\left(O_{K}\right) / \wp\left(W\left(O_{K}\right)\right)\right)=0\right\}$.

Another consequence of theorem 1.1 is the following:

Corollary 5.1. Let $u \geq 1$. Let a be a Witt vector of length $n$ and suppose that a lies in $W_{n}^{(u)}(K) \backslash W_{n}^{(u-1)}(K)$. Let $K_{a} / K$ denote the cyclic extension of degree $p^{k} \leq p^{n}$ defined by:

$$
K_{a}=K\left(\alpha_{0}, \ldots, \alpha_{n-1}\right)
$$


where $\alpha=\left(\alpha_{0}, \ldots, \alpha_{n-1}\right) \in W_{n}\left(K^{s e p}\right)$ is such that $\wp(\alpha)=a$. Then, the Artin conductor of $K_{a} / K$ is $\mathfrak{p}_{K}^{u+1}$.

In other words, according to proposition 5 of ([13], Chap.VI,§2), the highest non-trivial ramification group of $K_{a} / K$ is indexed by $u$. If $u=0$, then according to corollary 2.1 , the extension is unramified, i.e. the Artin conductor is trivial.

Proof. According to proposition 4.1, one may suppose that $a$ is reduced. Thus, its image modulo $\wp\left(W_{n}(K)\right)$ is in $W_{n}^{(u)} \bmod \wp\left(W_{n}(K)\right)$ but not in $W_{n}^{(u-1)} \bmod \wp\left(W_{n}(K)\right)$. Thereby, according to theorem $1.1, K_{a}$ is fixed by $G_{p^{n}}^{(u+1)}$ but not by $G_{p^{n}}^{(u)}$. Thus, since the Galois group of $K_{a} / K$ is a quotient of $G_{p^{n}}$, we conclude that its highest non-trivial ramification subgroup is indexed by $u$, according to the Herbrand's theorem.

For instance, if $a \in W_{n}(K)$ is the Witt vector $\left(T^{-1}, 0, \ldots, 0\right)$ then $M_{n}(a)=$ $p^{n-1}$ and $K_{a} / K$ is cyclic of degree $p^{n}$ since $T^{-1} \notin \wp(K)$. Thus, if $G$ denotes the Galois group of $K_{a} / K$ then $G^{\left(p^{n-1}\right)} \neq 1$ whereas $G^{\left(p^{n-1}+1\right)}=1$.

Likewise, we recover Theorem 1 of [2] in a natural way with more explicit arguments that avoid the Kato machinery. At the same time we render more precise Satz in part 3 of [12] by writing explicitly every crucial step of the proof and in particular by describing precisely the Schmid-Witt formula. Furthermore, we give a more general result that corresponds somehow to the projective limit of Schmid and Brylinski's results in the sense that we directly get the ramification groups for all maximal abelian extensions of exponent $p^{n}$ over $K$.

\section{Further remarks when the residue field is perfect}

This last section investigates the validity of the preceding computations when the residue field of $K$ is perfect. We shall give here partial answers: for the maximal abelian extension of exponent $p$ and for the inertia group of each maximal abelian extension of exponent $p^{n}$ over $K$.

The two main statements of this section are related to the following:

Proposition 6.1. If $x=\left(x_{0}, \ldots, x_{n-1}\right)$ is a Witt vector over $K$ whose components are all in $\mathfrak{p}_{K}$ then $x$ belongs to $\wp\left(W_{n}(K)\right)$.

Proof. Let $y=\sum_{i \geq 0} x^{p^{2}}$ : this sum converges in $W_{n}(K)$ with respect to the $p$-adic topology since it converges componentwise. We thus get the relation $x=\wp(-y)$ in $W_{n}(K)$. 
Clearly, it implies:

Corollary 6.1. Let $\left(x_{0}, \ldots, x_{n-1}\right)$ be a Witt vector in $W_{n}\left(O_{K}\right)$ and let $\pi_{0}, \ldots, \pi_{n-1}$ be $n$ elements in $\mathfrak{p}_{K}$. Then:

$$
\left(x_{0}+\pi_{0}, \ldots, x_{n-1}+\pi_{n-1}\right)-\left(x_{0}, \ldots, x_{n-1}\right) \in \wp\left(W_{n}\left(O_{K}\right)\right) .
$$

The first generalisation of theorem 1.1 to the case of a perfect residue field is concerned with the ramification groups of the maximal abelian extension of exponent $p$. It is theorem 1.2 of the introduction:

Theorem. Let $K$ be a local field of characteristic $p$ with perfect residue field. The Artin-Schreier isomorphism $\mathfrak{a s}_{1}$ induces isomorphisms of topological groups:

$$
G_{p}^{(0)} \stackrel{\simeq}{\longrightarrow} H_{p}^{(0)} \text { and } \quad G_{p}^{(u)} \stackrel{\simeq}{\longrightarrow} H_{p}^{(u-1)} \text { if } u \geq 1 .
$$

This theorem is based on the following crucial statement:

Proposition 6.2. Let $K$ be a local field and let $L$ and $L^{\prime}$ be two finite abelian extensions over $K$ with $L \cap L^{\prime}=K$, so that the compositum $L . L^{\prime}$ is Galois over $K$ of Galois group denoted by $G$. Let $H$ and $H^{\prime}$ be the subgroups of $G$ that fix $L$ and $L^{\prime}$ respectively. If $H$ is contained in the highest non trivial ramification subgroup of $G$ in the upper numbering, then for every integer $u \geq-1$ we have:

$$
G^{(u)} \simeq(G / H)^{(u)} \times\left(G / H^{\prime}\right)^{(u)} .
$$

Proof. Let us denote by $G^{(s)}$ the highest non-trivial ramification group of $G$, i.e. $G^{(s)} \neq\{1\}$ and $G^{(s+1)}=\{1\}$. By assumption: $H \subset G^{(s)}$.

Fix an integer $u \geq-1$. According to the Herbrand's theorem, we have:

$$
(G / H)^{(u)}=G^{(u)} H / H \text { and }\left(G / H^{\prime}\right)^{(u)}=G^{u} H^{\prime} / H .
$$

Therefore we can define a homomorphism:

$$
F_{u}:=G^{(u)} \longrightarrow(G / H)^{(u)} \times\left(G / H^{\prime}\right)^{(u)},
$$

which maps $\sigma \in G^{(u)}$ to $\left(\phi, \phi^{\prime}\right)$ where $\phi$ (resp. $\left.\phi^{\prime}\right)$ denotes the coset $\sigma H$ of $H$ in the group $G^{(u)} H$ (resp. the coset $\sigma H^{\prime}$ of $H^{\prime}$ in $G^{(u)} H^{\prime}$ ).

This map is injective because $H \cap H^{\prime}=\{1\}$ since $L \cap L^{\prime}=K$.

It is also surjective under the further condition that $H$ is contained in $G^{(s)}$. Indeed, if $u>s$, then $G^{(u)}=\{1\}$ and $F_{u}$ is obvioulsy surjective. Now if $u \leq s$ then $H$ is also a subgroup of $G^{(u)}$, and the ramification group $(G / \bar{H})^{(u)}$ is isomorphic to $G^{(u)} / H$. Therefore, considering an element 
$\left(\phi, \phi^{\prime}\right)$ in $(G / H)^{(u)} \times\left(G / H^{\prime}\right)^{(u)}$, there exist $\sigma$ in $G^{(u)}$ such that $\sigma=\phi$ $\bmod H$ and $\sigma^{\prime}$ in $G^{(u)} H^{\prime}$ such that $\sigma^{\prime}=\phi^{\prime} \bmod H^{\prime}$. But, by the natural isomorphism $G=H \times H^{\prime}$, there also exist $h \in H$ and $h^{\prime} \in H^{\prime}$ such that $\sigma h=\sigma^{\prime} h^{\prime}$. This means that $\sigma h$ in $G^{(u)}$ is mapped to $\left(\phi, \phi^{\prime}\right)$ by $F_{u}$, thereby proving the surjectivity of $F_{u}$ and our assertion.

Then, the proof of theorem 1.2 consists in a gradual process. Following proposition 2.1 of section 2, we first compute the ramification groups for a finite abelian extension of exponent $p$ over $K$. According to the ArtinSchreier-Witt theory, if $L$ is such an extension, it corresponds to the subgroup $B=\wp(L) \cap K$ of $K$ that contains $\wp(K)$ with finite index. Precisely, if $G_{B}$ is its Galois group, the isomorphism $\mathfrak{a s}_{1}$ induces an isomorphism $G_{B} \stackrel{\widetilde{\sim}}{\rightarrow} H_{B}$ where $H_{B}=\operatorname{Hom}\left(B / \wp(K), W_{1}\left(\mathbb{F}_{p}\right)\right)$.

Next, for each integer $u \geq-1$, we define the quotient group:

$$
B^{(v)}:=\left(\mathfrak{p}_{K}^{-v} \cap B+\wp(K)\right) / \wp(K),
$$

with $\mathfrak{p}_{K}^{0}=O_{K}$. In other words, $B^{(v)}$ is the image of $B \cap W_{1}^{(v)}(K)$ modulo $\wp(K)$ in the notation of section 1. By proposition 6.1 the first quotient $B^{(-1)}$ is trivial since $\mathfrak{p}_{K} \subset \wp(K)$. Moreover the quotient groups $B^{(u)}$ define an increasing filtration in $B / \wp(K)$ and since $B / \wp(K)$ is finite there exists $m \geq 0$ such that: $B^{(-1)}=\{1\} \subset B^{(0)} \subset B^{(1)} \subset \cdots \subset B^{(m)}=B / \wp(K)$.

Next, the groups:

$$
H_{B}^{(v)}:=\left\{\varphi \in H_{B}: \varphi\left(B^{(v)}\right)=0\right\}
$$

define a decreasing filtration in $H_{B}$ :

$$
\{1\}=H_{B}^{(m)} \subset \ldots \subset H_{B}^{(1)} \subset H_{B}^{(0)} \subset H_{B}^{(-1)}=H_{B} .
$$

We shall say that an integer $t \geq 0$ is a jump in the increasing filtration $B^{(v)}$ if $B^{(t-1)} \neq B^{(t)}$ and that an integer $t \geq-1$ is a jump in the filtration $H_{B}^{(v)}$ if $H_{B}^{(t)} \neq H_{B}^{(t+1)}$, i.e. if $t+1$ is a jump for the $B^{(v)}$ 's. Since $\kappa$ is perfect, it is easy to see that no jump $t \geq 1$ in the filtration $B^{(v)}$ is divisible by $p$. We then claim:

Proposition 6.3. The Artin-Schreier isomorphism $\mathfrak{a s}_{1}$ induces isomorphisms:

$$
G_{B}^{(0)} \stackrel{\simeq}{\longrightarrow} H_{B}^{(0)} \text { and } G_{B}^{(u)} \stackrel{\simeq}{\longrightarrow} H_{B}^{(u-1)} \text { for all } u \geq 1 .
$$

In particular, the Galois group $G_{B}$ has no jump which is divisible by $p$. 
Proof. For simplicity, let $G=G_{B}$. If $x$ is in $O_{L}$ let $\bar{x}$ be the image of $x$ modulo $\mathfrak{p}_{L}$.

First, by ramification theory there is a natural isomorphism:

$$
\begin{aligned}
& \psi_{1}: \quad G / G^{(0)} \stackrel{\simeq}{\longrightarrow} \operatorname{Gal}(l / \kappa) \\
& \sigma \bmod G^{(0)} \mapsto \bar{\sigma}:\left\{x \bmod \mathfrak{p}_{L} \mapsto \sigma(x) \bmod \mathfrak{p}_{L}\right\} .
\end{aligned}
$$

where $l / \kappa$ is the residue extension of $L / K$.

Then, the Artin-Schreier-Witt theory yields the isomorphism:

$$
\begin{aligned}
\psi_{2}: \operatorname{Gal}(l / \kappa) & \stackrel{\simeq}{ } \operatorname{Hom}\left(\wp(l) \cap \kappa / \wp(\kappa), W_{1}\left(\mathbb{F}_{p}\right)\right) \\
\bar{\sigma} & \mapsto \varphi_{\bar{\sigma}}:\{\bar{x} \bmod \wp(\kappa) \mapsto \bar{\sigma}(\bar{\xi})-\bar{\xi}\},
\end{aligned}
$$

for some $\bar{\xi}$ in $l$ such that $\bar{\xi}^{p}-\bar{\xi}=\bar{x}$ in $l$. Note that by the Hensel's lemma, $\bar{\sigma}(\bar{\xi})-\bar{\xi}=\sigma(\xi)-\xi \bmod \mathfrak{p}_{L}$, with $\sigma$ in $G$ such that $\psi_{1}\left(\sigma \bmod G^{(0)}\right)=\bar{\sigma}$.

Finally, since $B=\wp(L) \cap K, O_{K} \cap \wp(K)=\wp\left(O_{K}\right)$ and $\mathfrak{p}_{K} \subset \wp\left(O_{K}\right)$, there is a natural isomorphism $B^{(0)} \cong \wp(l) \cap \kappa / \wp(\kappa)$ that is induced by the projection map $O_{K} \rightarrow \kappa$. By duality, this gives rise to a third isomorphism:

$$
\begin{aligned}
\psi_{3}: \operatorname{Hom}\left(\wp(l) \cap \kappa / \wp(\kappa), W_{1}\left(\mathbb{F}_{p}\right)\right) & \stackrel{\simeq}{ }) \operatorname{Hom}\left(B^{(0)}, W_{1}\left(\mathbb{F}_{p}\right)\right) \\
\varphi & \mapsto \psi:\{x \bmod \wp(K) \mapsto \varphi(\bar{x})\} .
\end{aligned}
$$

Therefore, by composition we get the isomorphism:

$$
\begin{aligned}
\psi: \quad G / G^{(0)} & \stackrel{\simeq}{\longrightarrow} \operatorname{Hom}\left(B^{(0)}, W_{1}\left(\mathbb{F}_{p}\right)\right) \\
\sigma \bmod G^{(0)} & \mapsto \psi_{\sigma}:\{x \bmod \wp(K) \mapsto \sigma(\xi)-\xi\},
\end{aligned}
$$

for some $\xi \in L$ such that $\wp(\xi)=x$. Moreover, since $\psi_{2}$ is induced by $\mathfrak{a s}_{1}$, we get a commutative diagram with exact rows:

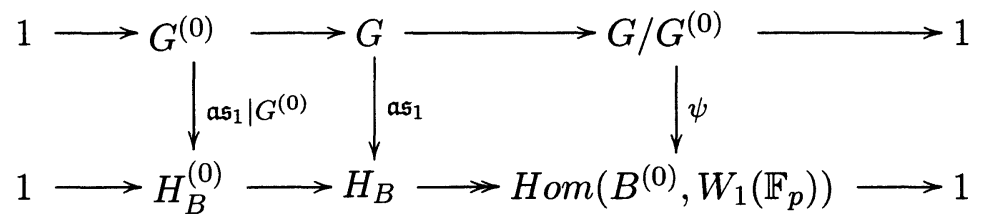

Thus $\mathfrak{a}_{1}$ induces an isomorphism $G^{(0)} \stackrel{\cong}{\rightrightarrows} H_{B}^{(0)}$, thereby proving the first assertion.

We then prove the isomorphisms $G^{(u)} \stackrel{\cong}{\rightrightarrows} H_{B}^{(u-1)}$ for $u \geq 1$ by induction on the order of the Galois group $G$, and more precisely on the exponent $n \geq 1$ where $|G|=p^{n}$. If $n=1$, this is proposition 2.1 of section 2 .

Now given an integer $n \geq 1$, we suppose that theorem 6.3 is true for any abelian extension over $\mathrm{K}$ of degree less than or equal to $p^{n}$ and let $L / K$ be an abelian extension of degree $p^{n+1}$. Therefore it has at most $n+1$ jumps in the filtration induced by its upper ramification groups. Let $G^{(s)}$ denote its highest non-trivial ramification group:

$$
G^{(s)} \neq 1, G^{(s+1)}=1 .
$$


Then $G^{(s)}$ is a $p$-group of order $p^{s} \leq p^{n+1}$; in particular it contains a cyclic subgroup $J_{1}$ of order $p$. Moreover there is another subgroup $J_{2}$ such that $G=J_{1} \times J_{2}$, and $J_{2}$ is of order at most $p^{n}$.

Let $M_{1}$ and $M_{2}$ denote the fixed subfields of $L / K$ under $J_{2}$ and $J_{1}$ respectively so that $M_{1} / K$ (resp. $M_{2} / K$ ) has Galois group $G / J_{1}$ (resp. $\left.G / J_{2}\right)$. Then we simultaneously get:

$$
M_{1} \cap M_{2}=K \text { and } L=M_{1} \cdot M_{2} .
$$

Therefore, according to proposition 6.2 we get for every integer $u \geq 1$ :

$$
G^{(u)} \simeq\left(G / J_{1}\right)^{(u)} \times\left(G / J_{2}\right)^{(u)} .
$$

We then denote by $A_{1}$ (resp. $\left.A_{2}\right)$ the subgroup $\wp\left(M_{1}\right) \cap K\left(\right.$ resp. $\left.\wp\left(M_{2}\right) \cap K\right)$ of $B$. Since the extensions $M_{1}$ and $M_{2}$ are of degree at most $p^{n}$ over $K$, we get the following isomorphism of topological groups for each integer $u \geq 1$ :

$$
G^{(u)} \cong H_{A_{1}}^{(u-1)} \times H_{A_{2}}^{(u-1)}
$$

given by $\sigma \mapsto\left(\mathfrak{a s}_{1}\left(\sigma_{\mid M_{1}}\right), \mathfrak{a s}_{1}\left(\sigma_{\mid M_{2}}\right)\right)$.

On the other hand, by Galois theory, the group $G$ is isomorphic to the product $G / J_{1} \times G / J_{2}$ in a natural way. Applying the Artin-Schreier-Witt isomorphism $\mathfrak{a s}_{1}$ it then results the isomorphism:

$$
\mathcal{E}:=H_{B} \stackrel{\simeq}{\longrightarrow} H_{A_{1}} \times H_{A_{2}} \text {. }
$$

For all $u \geq 1$, we claim that under $\mathcal{E}$ the subgroup $H_{B}^{(u-1)}$ is sent onto:

$$
\mathcal{E}\left(H_{B}^{(u-1)}\right)=H_{A_{1}}^{(u-1)} \times H_{A_{2}}^{(u-1)}
$$

Indeed, the first inclusion is trivial since $A_{i}^{(u-1)}=B^{(u-1)} \cap A_{i} / \wp(K)$, for $i=1,2$.

Conversely, let $\left(\sigma_{1}, \sigma_{2}\right)$ be in $H_{A_{1}}^{(u-1)} \times H_{A_{2}}^{(u-1)}$. By the isomorphism $\mathcal{E}$, there exists $f$ in $H_{B}$ such that $f_{\mid A_{i} / \wp(K)}=\sigma_{i}$ for $i=1,2$. In particular, $f\left(B^{(u-1)} \cap A_{i} / \wp(K)\right)=0$.

Now, by duality, $\mathcal{E}$ induces the isomorphism:

$$
B / \wp(K) \stackrel{\simeq}{\longrightarrow} A_{1} / \wp(K) \times A_{2} / \wp(K),
$$

and so $f$ is zero on $B^{(u-1)}$, which means that $f$ lies in $H_{B}^{(u-1)}$, thereby proving the other isomorphism of topological groups:

$$
\forall u \geq 1, H_{B}^{(u-1)} \stackrel{\simeq}{\longrightarrow} H_{A_{1}}^{(u-1)} \times H_{A_{2}}^{(u-1)}
$$

Consequently, by composition, the Artin-Schreier map induces the isomorphism of topological groups:

$$
\forall u \geq 1, G^{(u)} \stackrel{\simeq}{\longrightarrow} H_{B}^{(u-1)},
$$

which completes the proof of proposition 6.3 . 
Therefore, by taking the inverse limit on all finite abelian extensions of exponent $p$, we finally prove theorem 1.2 :

Proof. Let $\mathcal{B}$ denote the set of all subgroups of $K$ that contain $\wp(K)$ with finite index. If $B$ is such a group, we denote by $G_{B}$ the Galois group of the corresponding finite abelian extension of exponent $p$ over $K$ that is the compositum of all extensions $K(\alpha) / K$ with $\alpha \in K^{\text {sep }}$ and $\wp(\alpha) \in B$. According to proposition 6.3 , there is an isomorphism $G_{B}^{(0)} \stackrel{\simeq}{\longrightarrow} H_{B}^{(0)}$, for every $B$ in $\mathcal{B}$. Let $\psi_{B}$ denote this isomorphism.

On the other hand, we have by definition:

$$
G_{p}^{(0)}=\lim _{\longleftarrow} G_{B}^{(0)},
$$

when $B$ runs over $\mathcal{B}$ and with respect to the restriction homomorphisms as transition maps. Besides, we also get:

$$
H_{p}^{(0)} \simeq \lim _{\longleftarrow}^{(0)},
$$

since $\left(K \cap \mathfrak{p}_{K}^{0}\right) / \wp(K)$ is the union of all $B^{(0)}$ for $B \in \mathcal{B}$.

Then, for all $B \subset B^{\prime}$ in $\mathcal{B}$ the following diagram is commutative:

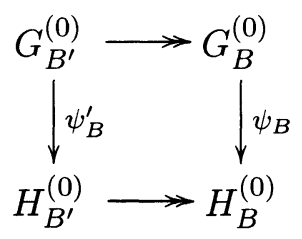

where the horizontal maps are the restriction maps.

Therefore, by the universal property of the inverse limit and since all groups $G_{B}^{(0)}$ and $H_{B}^{(0)}$ are compact, there exists a unique isomorphism of topological groups:

and it is induced by $\mathfrak{a s}_{1}$.

$$
G_{p}^{(0)} \stackrel{\simeq}{\longrightarrow} H_{p}^{(0)}
$$

For $u \geq 1$, the proof is roughly the same once we replace the index 0 by $u$ for $G_{p}$ and by $u-1$ for $H_{p}$.

As a corollary, we shall mention:

Corollary 6.2. No jump in the filtration of the ramification subgroups of $G_{p}$ is divisible by $p$.

When $K$ has perfect residue field, another attempt is made to generalise theorem 1.1 with a description of the inertia group of each maximal 
abelian extension of exponent $p^{n}$ over $K$. This is theorem 1.3 stated in the introduction:

Theorem. Let $K$ be a local field of characteristic $p$ with perfect residue field. For each $n \geq 1$, the Artin-Schreier-Witt isomorphism $\mathfrak{a s}_{n}$ induces on the inertia group of $G_{p^{n}}$ the isomorphism of topological groups:

$$
G_{p^{n}}^{(0)} \stackrel{\simeq}{\longrightarrow}\left\{\varphi \in H_{p^{n}} ; \varphi\left(W_{n}\left(O_{K}\right) / \wp\left(W_{n}\left(O_{K}\right)\right)\right)=0\right\} .
$$

Passing to the inverse limit, $\mathfrak{a s}_{\infty}$ yields an isomorphism of topological groups:

$$
G_{p^{\infty}}^{(0)} \stackrel{\simeq}{\longrightarrow}\left\{\varphi \in H_{p^{\infty}} ; \varphi\left(W\left(O_{K}\right) / \wp\left(W\left(O_{K}\right)\right)\right)=0\right\} .
$$

One proof for the first assertion of this theorem is the following:

Proof. Let $K_{n}$ be the maximal abelian extension of exponent $p^{n}$ over $K$. Its residue extension is the maximal abelian extension $\kappa_{n}$ of exponent $p^{n}$ over $\kappa$, hence the isomorphism $\operatorname{Gal}\left(\kappa_{n} / \kappa\right) \stackrel{\simeq}{\longrightarrow} \bar{h}_{p^{n}}$, where $\bar{h}_{p^{n}}$ denotes the group $\operatorname{Hom}\left(W_{n}(\kappa) / \wp\left(W_{n}(\kappa)\right), W_{n}\left(\mathbb{F}_{p}\right)\right)$. From corollary 6.1 one may define a homomorphism $\phi_{n}: H_{p^{n}} \longrightarrow \bar{h}_{p^{n}}$ by:

$$
\phi_{n}(\varphi):=\left\{\left(\bar{x}_{0}, \ldots, \bar{x}_{n-1}\right)+\wp\left(W_{n}(\kappa)\right) \mapsto \varphi\left(\left(x_{0}, \ldots, x_{n-1}\right)+\wp\left(W_{n}(K)\right)\right)\right\},
$$

where $\bar{x}_{i}$ is the image of $x_{i} \in O_{K}$ modulo $\mathfrak{p}_{K}$.

Let $\mathfrak{K}_{n}$ be the kernel of $\phi_{n}$. The interplay between ramification theory and Artin-Schreier-Witt theory gives the following commutative diagram:

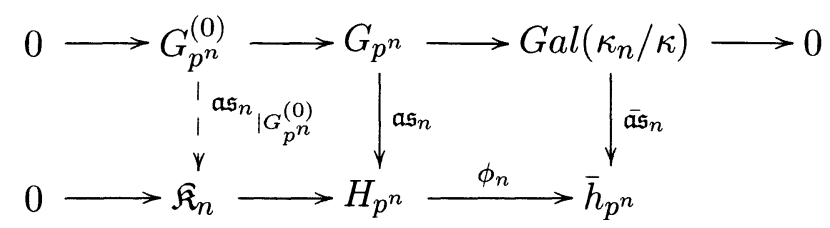

where the two right hand vertical maps are isomorphisms.

This yields an isomorphism $G_{p^{n}}^{(0)} \stackrel{\cong}{\rightrightarrows} \mathfrak{K}_{n}$.

Since $\wp\left(W_{n}\left(O_{K}\right)\right)=\wp\left(W_{n}(K)\right) \cap W_{n}\left(O_{K}\right)$ it follows that:

$$
G_{p^{n}}^{(0)} \stackrel{\cong}{\rightrightarrows}\left\{\varphi \in H_{p^{n}}: \varphi\left(W_{n}\left(O_{K}\right) / \wp\left(W_{n}\left(O_{K}\right)\right)\right)=0\right\} .
$$

Now, these two former groups are compact. Besides, this isomorphism is continuous since it is induced by the Artin-Schreier-Witt isomorphism $\mathfrak{a s}_{n}$, thus it is also a homeomorphism as was to be shown.

Passing to the inverse limit, we then get the second assertion of theorem 1.3 whose proof is based on the following identification: 
Lemma 6.1. For $n \geq 1$, there is a natural isomorphism of topological groups:

$$
H_{p^{n}} \stackrel{\simeq}{\longrightarrow} \operatorname{Hom}\left(W(K) / \wp(W(K)), W_{n}\left(\mathbb{F}_{p}\right)\right),
$$

given by $\varphi \mapsto\left\{x+\wp(W(K)) \mapsto \varphi\left(t_{n}(x)+\wp\left(W_{n}(K)\right)\right)\right\}$, where $t_{n}: W(K) \rightarrow$ $W_{n}(K)$ is the truncation map.

Proof. Let $\mathcal{V}(K)$ denote the quotient group $W(K) / \wp(K)$. We first claim the existence of an isomorphism:

$$
\Theta_{n}: W_{n}(K) / \wp\left(W_{n}(K)\right) \stackrel{\simeq}{\longrightarrow} \mathcal{V}(K) / \mathbf{p}^{n} \mathcal{V}(K),
$$

given by $\left(x_{0}, \ldots, x_{n-1}\right)+\wp\left(W_{n}(K)\right) \mapsto(x+\wp(W(K))) \bmod \mathbf{p}^{n} \mathcal{V}$, for some Witt vector $x \in W(K)$ such that $t_{n}(x)=\left(x_{0}, \ldots, x_{n-1}\right)$.

Indeed, following the relation $V \circ F=F \circ V=\mathbf{p}$, this is a consequence of:

$$
\mathbf{p}^{n} W(K)+\wp(W(K))=V^{n}(W(K))+\wp(W(K)),
$$

since $W_{n}(K) \simeq W(K) / V^{n} W(K)$ (see section 2).

Therefore, by duality, we get an additive isomorphism:

$$
H_{p^{n}} \stackrel{\simeq}{\longrightarrow} \operatorname{Hom}\left(\mathcal{V}(K) / \mathbf{p}^{n} \mathcal{V}(K), W_{n}\left(\mathbb{F}_{p}\right)\right)
$$

given by $f \mapsto f \circ \Theta_{n}^{-1}$.

Now since $W_{n}\left(\mathbb{F}_{p}\right)$ is annihilated by $\mathbf{p}^{\mathbf{n}}$, it gives rise to the isomorphism:

$$
\Phi_{n}:=H_{p^{n}} \stackrel{\simeq}{\longrightarrow} \operatorname{Hom}\left(\mathcal{V}(K), W_{n}\left(\mathbb{F}_{p}\right)\right) .
$$

Moreover, all the maps $\varphi_{a} \circ \Phi_{n}$ for $a \in \mathcal{V}(K)$ are continuous, where $\varphi_{a}$ is the natural projection $\operatorname{Hom}\left(\mathcal{V}(K), W_{n}\left(\mathbb{F}_{p}\right)\right) \rightarrow W_{n}\left(\mathbb{F}_{p}\right)$ that sends $h$ to $h(a)$. Therefore, $\Phi_{n}$ is continuous and it is a homeomorphism since both $H_{p^{n}}$ and $\operatorname{Hom}\left(\mathcal{V}(K), W_{n}\left(\mathbb{F}_{p}\right)\right)$ are compact groups, as was to be shown.

Whence the end of the proof for theorem 1.3:

Proof. For all integers $n \geq m$, let $\pi_{n m}$ denote the natural surjection map:

$$
\begin{aligned}
\pi_{n m}: H_{p^{n}} & \rightarrow H_{p^{m}} \\
\varphi & \mapsto \varphi \bmod V^{m} W\left(\mathbb{F}_{p}\right) .
\end{aligned}
$$

under the identification of lemma 6.1. The system $\left\{H_{p^{n}}, \pi_{n m}\right\}_{n}$ is projective and its inverse limit is equal to $H_{p^{\infty}}$.

Besides, the identification of lemma 6.1 leads to other isomorphisms:

$$
H_{p^{n}}^{(0)} \stackrel{\simeq}{\longrightarrow}\left\{\varphi \in H_{p^{n}}: \varphi\left(W\left(O_{K}\right) / \wp\left(W\left(O_{K}\right)\right)\right)=0\right\},
$$

that commute with the isomorphisms of theorem 1.3.

We denote by $H_{p^{\infty}}^{(0)}$ the compact group :

$$
H_{p^{\infty}}^{(0)} \stackrel{\simeq}{\longrightarrow}\left\{\varphi \in H_{p^{\infty}}: \varphi\left(W\left(O_{K}\right) / \wp\left(W\left(O_{K}\right)\right)\right)=0\right\} .
$$


The projection maps $\pi_{n}: H_{p^{\infty}} \rightarrow H_{p^{n}}$, given by :

$$
\pi_{n}: \varphi \mapsto \varphi \bmod V^{n} W\left(\mathbb{F}_{p}\right),
$$

are all compatible with the projective system $\left\{H_{p^{n}}, \pi_{n m}\right\}_{n}$. Thus, according to $\left([8]\right.$, cor. 1.1.8, a.): $H_{p^{\infty}}^{(0)}=\lim _{\leftarrow} \pi_{n}\left(H_{p^{\infty}}^{(0)}\right)$.

But, for each $n \geq 1, \pi_{n}\left(\overleftarrow{H_{p^{\infty}}^{(0)}}\right) \subset H_{p^{n}}^{(0)}$, hence the inclusion $H_{p^{\infty}}^{(0)} \subset$ $\underset{\lim }{\longleftarrow} H_{p^{n}}^{(0)}$.

Conversely, let $\varphi$ be a homomorphism in $H_{p^{\infty}}$. We get:

$$
\begin{aligned}
\varphi \in \lim _{\longleftarrow} H_{p^{n}}^{(0)} & \Longrightarrow \varphi\left(W\left(O_{K}\right) / \wp\left(W\left(O_{K}\right)\right)\right) \in \cap_{n} V^{n} W\left(\mathbb{F}_{p}\right) \\
& \Longrightarrow \varphi\left(W\left(O_{K}\right) / \wp\left(W\left(O_{K}\right)\right)\right)=0 \text { in } W\left(\mathbb{F}_{p}\right) \\
& \Longrightarrow \varphi \in H_{p^{\infty}}^{(0)},
\end{aligned}
$$

thereby proving $H_{p^{\infty}}^{(0)}=\lim H_{p^{n}}^{(0)}$.

Now, for all integers $n \geq m$, the following diagram is commutative:

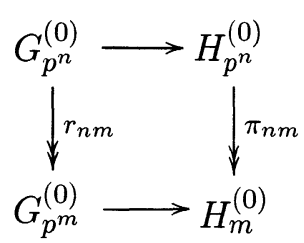

where the horizontal maps are induced by the Artin-Schreier-Witt isomorphisms $\mathfrak{a s}_{n}$ and $\mathfrak{a} \mathfrak{s}_{m}$ respectively and where $r_{n m}: G_{p^{n}}^{(0)} \rightarrow G_{p^{m}}^{(0)}$ is the restriction map.

Therefore, since all groups $G_{p^{n}}^{(0)}$ and $H_{p^{n}}^{(0)}$ are compact, we obtain an isomorphism of topological groups $\lim G_{p^{n}}^{(0)} \stackrel{\simeq}{\leftrightarrows} \lim H_{p^{n}}^{(0)}$ and thus $G_{p^{\infty}}^{(0)} \stackrel{\cong}{\rightrightarrows}$ $H_{p^{\infty}}^{(0)}$. Besides, by construction, this isomorphism is induced by the ArtinSchreier-Witt isomorphism $\mathfrak{a s}_{\infty}$, which completes the proof of theorem 1.3.

\section{References}

[1] E. Artin, O.SchreIER, Eine Kennzeichnung der reell algeschlossenen Körper. Abh. Math. Sem. Hamburg 5 (1927), 225-231.

[2] J.-L. BRYLINSKI, Théorie du corps de classes de Kato et revêtements abéliens de surfaces. Ann. Inst. Fourier, 33.3, Grenoble (1983), 23-38.

[3] I.B. Fesenko, S.V. Vostokov, Local Fields and Their Extensions. Translation of Mathematical Monographs 121, Amer. Math. Soc. (1993).

[4] M. Garuti, Linear sytems attached to cyclic inertia. (Berkeley, CA, 1999), 377-386, Proc. Sympos. Pure Math., 70, Amer. Math. Soc., Providence, RI (2002). 
[5] H. HASSE, Theorie der relativ-zyklischen algebraischen Funktionenkörper, insbesondere bei endlichem Konstantenkörper. J. Reine Angew. Math. 172 (1934), 37-54.

[6] M. Hazewinkel, Abelian extensions of local fields, Ph.D. thesis. Universiteit Nijmegen, Holland (1969).

[7] E. MAus, Die Gruppentheoretische Struktur der Verzweigungsgruppenreihen. J. Reine. Angew. Math. 230 (1968), 1-28.

[8] L. Ribes, P. ZalesskiI, Profinite groups. A series of Modern Surveys in Mathematics, Volume 40, Springer (2000).

[9] P. Roquette, Class Field Theory in characteristic $p$. Its origin and development. K. Miyake (ed.), Class Field Theory- Its Centenary and Prospect, Advanced Studies In Pure Mathematics, vol. 30, Tokyo (2000), 549-631.

[10] H.L. SCHMID, Über das Reziprozitätsgezsetz in relativ-zyklischen algebraischen Funktionkörpern mit endlichem Konstantenkörper. Math. Z. 40 (1936), no. 1, 94-109.

[11] H.L. ScHmID, Zyklischen algebraische Funktionkörper vom Grade $p^{n}$ über endlichem Konstantenkörper der Charakteristik p. J. Reine Angew. Math. 175, (1936), 108-123.

[12] H.L. SCHMID, Zur Arithmetik der zyklischen p-Körper. J. Reine Angew. Math. 176 (1937), 161-167

[13] J.-P. Serre, Corps Locaux. Hermann, Paris (1962). [English translation : Local Fields. Graduate Texts in Math. 67, Springer, New York (1979)].

[14] S.S. Shatz, Profinite groups, arithmetic and geometry. Princeton university press and university of Tokyo press (1972).

[15] O. Teichmüller, Zerfallende zyklische p-Algebren. J. Reine Angew. Math., 174 (1936), 157-160.

[16] L. Thомаs, Arithmétique des extensions d'Artin-Schreier-Witt, Ph.D. (2005). Université Toulouse II Le Mirail.

[17] E. WiTT, Zyklische Körper und Algebren der Charakteristik vom Grad $p^{n}$. J. Reine Angew. Math., 174 (1936), 126-140.

Lara Thomas

Equipe GRIMM Chaire de Structures Algébriques et Géométriques

Université Toulouse II Ecole Polytechnique Fédérale de Lausanne

5, allées A. Machado SB - IMB (Bâtiment MA) Station 8

31058 Toulouse, France $\mathrm{CH}-1015$ Lausanne

E-mail : 1thomas@univ-tlse2.fr

$U R L:$ http://alg-geo.epfl. ch/ l thomas 